\title{
Developmental chromatin programs determine oncogenic competence in
}

\section{melanoma}

Arianna Baggiolini ${ }^{1} \uparrow$, Scott J. Callahan ${ }^{2} \uparrow$, Tuan Trieu³ ${ }^{3}$, Mohita M. Tagore ${ }^{4}$, Emily Montal ${ }^{4}$, Joshua M. Weiss ${ }^{5}$, Sam E. Tischfield ${ }^{6}$, Yujie Fan ${ }^{7}$, Nathaniel R. Campbell ${ }^{5}$, Nathalie Saurat ${ }^{1}$,

Travis Hollmann ${ }^{8}$, Theresa Simon-Vermot ${ }^{4}$, Satish K. Tickoo ${ }^{8}$, Barry S. Taylor ${ }^{6}$, Richard Koche $^{9}$, Ekta Khurana ${ }^{3}$, Lorenz Studer ${ }^{1 *}$, Richard M. White ${ }^{10 *}$.

${ }^{1}$ Developmental Biology, The Center for Stem Cell Biology, Memorial Sloan Kettering Cancer Center, New York, NY 10065, USA.

${ }^{2}$ Developmental Biology, The Center for Stem Cell Biology, Memorial Sloan Kettering Cancer Center, New York, NY 10065, USA; Memorial Sloan Kettering Cancer Center, Cancer Biology and Genetics and Department of Medicine, New York, NY 10065, USA; Memorial Sloan Kettering Cancer Center, Gerstner Graduate School of Biomedical Sciences, New York, NY 10065, USA.

${ }^{3}$ Institute for Computational Biomedicine, Weill Cornell Medical College, New York, NY 10021, USA; Department of Physiology and Biophysics, Weill Cornell Medicine, 1300 York Avenue, New York, NY 10065, USA; Caryl and Israel Englander Institute for Precision Medicine, Weill Cornell Medicine, New York, NY, USA; Meyer Cancer Center, Weill Cornell Medicine, New York, NY, USA.

${ }^{4}$ Memorial Sloan Kettering Cancer Center, Cancer Biology and Genetics and Department of Medicine, New York, NY 10065, USA. 
${ }^{5}$ Memorial Sloan Kettering Cancer Center, Cancer Biology and Genetics and Department of Medicine, New York, NY 10065, USA; Weill Cornell/Rockefeller/Sloan-Kettering TriInstitutional MD-PhD Program, New York, NY 10065, USA.

${ }^{6}$ Department of Epidemiology and Biostatistics, Memorial Sloan Kettering Cancer Center, New York, NY 10065, USA.

${ }^{7}$ Developmental Biology, The Center for Stem Cell Biology, Memorial Sloan Kettering Cancer Center, New York, NY 10065, USA; Weill Graduate School of Medical Sciences of Cornell University, New York, NY 10065, USA.

${ }^{8}$ Department of Pathology, Memorial Sloan Kettering Cancer Center, New York, NY 10065, USA.

${ }^{9}$ Center for Epigenetics Research, Memorial Sloan Kettering Cancer Center, New York, NY 10065, USA.

${ }^{10}$ Memorial Sloan Kettering Cancer Center, Cancer Biology and Genetics and Department of Medicine, New York, NY 10065, USA; Weill Cornell Medical College, New York, NY 10065, USA.

$\uparrow$ These authors contributed equally

*Co-corresponding and equally contributing authors. Email: studerl@mskcc.org and whiter@mskcc.org 


\begin{abstract}
:
Oncogenes are only transforming in certain cellular contexts, a phenomenon called oncogenic competence. The mechanisms regulating this competence remain poorly understood. Here, using a combination of a novel human pluripotent stem cell (hPSC)-based cancer model along with zebrafish transgenesis, we demonstrate that the transforming ability of BRAF ${ }^{\mathrm{V} 600 \mathrm{E}}$ depends upon the intrinsic transcriptional program present in the cell of origin. Remarkably, in both systems, melanocytes (MC) are largely resistant to BRAF. In contrast, both neural crest (NC) and melanoblast $(\mathrm{MB})$ populations are readily transformed. Molecular profiling reveals that NC/MB cells have markedly higher expression of chromatin modifying enzymes, and we discovered that the chromatin remodeler ATAD2 is required for response to BRAF and tumor initiation. ATAD2 forms a complex with SOX10, allowing for expression of downstream oncogenic programs. These data suggest that oncogenic competence is mediated by developmental regulation of chromatin factors, which then allow for proper response to those oncogenes.
\end{abstract}

\title{
Main Text:
}

Alterations to DNA structure, including mutations, copy number variations and gene fusions are typically considered initiating events in most human cancers (1). However, these alterations are layered onto existing transcriptional programs in the cell of origin. The importance of these preexisting cellular lineage programs is highlighted by the fact that certain DNA mutations are only tumorigenic in certain cell types $(2,3)$, which we refer to as oncogenic competence.

In melanoma, it has been shown that tumor initiation by $\mathrm{BRAF}^{\mathrm{V} 600 \mathrm{E}}$ activates a neural crest lineage program $(4,5,6,7)$. In particular crestin, a gene that is specifically expressed in neural 
crest cells and is downregulated later during embryonic development, is reactivated in melanoma initiating cells and then maintained in the tumor $(6)$. The activation of NC-lineage specific mechanisms (4-10) together with oncogene mutations such as $\mathrm{BRAF}^{\mathrm{V} 600 \mathrm{E}}$ are fundamental for the acquisition of a malignant state $(11,12)$. However, it is not known why a NC-like state is required and particularly susceptible to oncogenic transformation by BRAF and what the factors are regulating this state.

Lineage programs are centrally intertwined with the cell of origin. Within a developmental lineage, cells can exist along a wide spectrum of differentiation states. After emergence from the neural crest, migrating neural crest progenitors give rise to populations of melanoblasts, melanocyte stem cells, or differentiated melanocytes. Which cell along that spectrum is intrinsically capable of giving rise to melanoma even above and beyond the influence of extrinsic microenvironmental factors has been a matter of debate $(13,14,15)$. Here, we asked what mechanisms define melanoma competence and how it is regulated.

\section{Zebrafish models show that $\mathrm{NC}$ and $\mathrm{MB}$, but not $\mathrm{MC}$, are oncogenic competent}

The melanocyte lineage develops as a hierarchy of cells that start as undifferentiated neural crest cells, proceeding through a melanoblast stage and then finally differentiating into mature, pigmented melanocytes. To understand which cells within this lineage are most sensitive to an oncogenic insult, we engineered zebrafish to initiate tumors in either neural crest (NC), melanoblasts $(\mathrm{MB})$ or melanocytes $(\mathrm{MC})$ by using stage-specific promoters to drive BRAF ${ }^{\mathrm{V} 600 \mathrm{E}}$. We used the sox 10 promoter to drive $\mathrm{BRAF}^{\mathrm{V} 600 \mathrm{E}}$ expression in the $\mathrm{NC}$, the mitfa promoter to drive it in the MB and the tyrpl promoter to drive it in the MC. These transgenic constructs were injected into 1-cell embryos of a $\mathrm{p} 53^{-/-}$deficient background, and the animals were allowed to 
grow up to adulthood to monitor for tumor formation (Fig. 1A). We found that animals that expressed the $\mathrm{BRAF}^{\mathrm{V} 600 \mathrm{E}}$ oncogene either in the $\mathrm{NC}$ cells or the $\mathrm{MB}$ developed aggressive tumors (Fig. 1B, 1C, 1D, fig. S1A and S1B) with nearly $100 \%$ penetrance in the NC lineage (Fig. 1B). Surprisingly, the tyrp1:BRAF ${ }^{\mathrm{V} 600 \mathrm{E}} ; \mathrm{p} 53^{-/-}$transgenic animals failed to develop tumors, but instead developed small patches of nevus-like cells (Fig. 1B, 1E, 1H, 1K and fig. S1C). We analyzed both the NC- and the MB-derived tumors and found that they stained equally for pERK (Fig. 1I, 1J), indicating that the BRAF pathway (Fig. 1F, 1G) was being activated. H\&E showed that the NC- and MB-derived tumors were histologically distinct (fig. S1A, S1B). The NCderived tumors appeared undifferentiated and with little melanin. Immunohistochemistry showed that the NC-derived tumors were predominantly positive for the neuronal markers huc/hud and ncam and sparsely for sox10 (Fig. 1L, 1N, 1P), likely reflecting the multipotency of the NC (16, $17,18)$ and resembling reports of melanoma with neuronal type differentiation $(19,20)$. On the contrary, the MB-derived tumors had an appearance characteristic of typical cutaneous melanoma, with numerous pigmented areas, and they stained positive for not only sox 10 (Fig. 1M), as previously shown for most melanomas $(8,21)$, but also positive for mlana (Fig. 1Q), while being negative for the neuronal markers huc/hud (Fig. 1O). To further confirm the differences between the NC- and MB-derived tumors, we performed RNA-seq and found that these tumors clustered separately from each other by PCA and hierarchical clustering (Fig. 1R, fig. S1D and table S1). Examination of specific pathways in each tumor revealed that, consistent with our immunohistochemistry, the NC-derived tumors expressed neuronal genes while the MB-derived melanomas expressed genes related to the melanocytic lineage (fig. S1E). We performed Gene Set Association Analysis (GSAA) and confirmed that NC-derived tumors displayed a strong neuronal phenotype and a gene signature that characterizes poor survival in 
neuroblastoma (fig. S1F) (22). These data suggest that competence to respond to BRAF ${ }^{\mathrm{V} 600 \mathrm{E}}$ is biased towards cells of origin exhibiting either a NC or MB gene program, that these give rise to distinct tumors, and that MC are relatively resistant to this insult.

\section{A hPSC-based cancer model recapitulates the zebrafish models}

Because mouse studies routinely use MC promoters to efficiently drive melanoma (i.e. the tyrCre line, 23), this raised the question of whether the above findings were unique to the zebrafish system. To interrogate this, we developed a novel human cancer paradigm based on the use of hPSCs to model oncogenic competence for melanoma in a manner similar to the zebrafish studies. We previously demonstrated that hPSCs can be progressively differentiated into NC cells, MB or MC (24). We used gene targeting in hPSCs to introduce oncogenic BRAF ${ }^{\mathrm{V} 600 \mathrm{E}}$ and to inactivate the tumor suppressors RB1, TP53 and P16 (referred to hereafter as 3xKO cells) (Fig. 2A). These 3xKO engineered cells were then differentiated into NC cells, MB and mature $\mathrm{MC}(24,25)$ (Fig. 2A and fig. S2A, S2B) and BRAF ${ }^{\mathrm{V} 600 \mathrm{E}}$ induced by doxycycline (fig. S2L). Western blot analysis for pERK demonstrated equal activation of the BRAF pathway across all three cell types (Fig. 2B), similar to the results in the zebrafish studies above. To test their tumorigenic capacity, we transplanted each of three cell types subcutaneously in immunodeficient NOD-scid IL2Rgamma ${ }^{\text {null }}$ (NSG) mice, a model that has been used before to assess the tumorigenic potential of the cells $(26,27)$, and the mice were exposed either to a doxcontaining or to a normal diet. Similar to the zebrafish, we found that both NC cells and MB expressing $\mathrm{BRAF}^{\mathrm{V} 600 \mathrm{E}}$ in $3 \mathrm{xKO}$ background readily formed tumors in the mice (Fig. 2C, 2D), but that the $3 \mathrm{xKO} \mathrm{MC}$ largely failed to do so, with only a single animal developing a tumor under this condition (Fig. 2E). Residual cells from the site of MC transplant at day 197 post 
injection showed features of a benign nevus-like structure consistent with them becoming senescent over time (fig. S2K). As a control, we also transplanted wildtype (WT) NC cells, MB and $\mathrm{MC}$ and found that these were unable to grow in vivo, as expected (data not shown). We performed histological analysis of the NC- and MB-derived tumors (Fig. 2F-S and fig. S2C-J) and found high level expression of $\mathrm{BRAF}^{\mathrm{V} 600 \mathrm{E}}$ and pERK in both (Fig. 2F-2I). Analogous to the zebrafish tumors, the human PSC-based 3xKO NC cells gave rise to tumors that showed a strong preponderance of neuronal markers (Fig. 2P, 2R), whereas the 3xKO MB were positive for all the common markers of melanoma and were pathologically categorized as desmoplastic melanomas (Fig. 2K, 2M, 2O). We performed Gene Set Enrichment Analysis (GSEA), and we found that NC cells were transcriptionally similar to the NC-derived tumors in the fish (Figure $\mathrm{S} 3 \mathrm{~B}$ ) and that MB were transcriptionally similar to the MB-derived tumors in the fish (fig. S3C), which further corroborated the comparability between the zebrafish and the hPSC-derived cancer models. To ensure that our hPSC-derived tumors are relevant to human patients, we performed RNA-seq of the BRAF ${ }^{\mathrm{V} 600 \mathrm{E}} 3 \mathrm{xKO} \mathrm{NC}$ cells, $\mathrm{MB}$ and $\mathrm{MC}$ and compared their expression profiles to data from The Cancer Genome Atlas (TCGA), using a published signature for melanoma subgroups (28). Strikingly, we observed that the hPSC-derived 3xKO dox NC cells and 3xKO dox MB strongly clustered with the human melanoma patient samples (Fig. 2T), whereas the hPSC-derived 3xKO dox MC did not. Interestingly, we unexpectedly found that the 3xKO NC cells and $3 \times \mathrm{XO} \mathrm{MB}$, even without $\mathrm{BRAF}^{\mathrm{V} 600 \mathrm{E}}$ induction, could form tumors in mice indicating that loss of tumor suppressors alone gives these cells enough of a proliferative advantage to grow in this context. As mentioned, we did find a single animal that grew a tumor after transplant with $3 \mathrm{xKO} \mathrm{MC}$, but exome sequencing showed that this single tumor had gained copy number alterations in $\mathrm{MYC}\left(\right.$ padj=2.30 $\left.\mathrm{E}^{-15}\right)$ (data not shown), among other genomic changes, likely 
contributing to its capacity to grow in vivo. Thus, similar to what we found in the zebrafish, tumorigenic capacity in our novel human melanoma model is strongly biased towards NC or MB lineage, and that $\mathrm{MC}$ are relatively refractory to tumorigenesis although can be coaxed to do so with additional genetic lesions.

\section{$\mathrm{NC} / \mathrm{MB}$ cells have strong transcriptional responses to BRAF, but MC have little, despite comparable activation of pERK}

To gain insight into why these cells differed in oncogenic competence, we performed RNA-seq of $\mathrm{NC}, \mathrm{MB}$ and $\mathrm{MC}$ cells $\pm \mathrm{BRAF}^{\mathrm{V} 600 \mathrm{E}}$ on both the WT and 3xKO background. We observed that dox-induced $\mathrm{BRAF}^{\mathrm{V} 600 \mathrm{E}}$ expression caused dramatic transcriptional changes in both the NC and MB (Fig. 2U, 2V, 2W, 2X, fig. S3A and S3D). In contrast, the transcriptional response to BRAF in MC was nearly absent, with only few genes being altered (23 genes upregulated (padj < 0.01$)$ and 114 downregulated (padj < 0.01)) (Fig. 2Y, 2Z, fig. S3A, S3D and table S2). Thus, despite equally robust activation of pERK across all three cell types (as above in Fig. 2B), this indicates that the MC state was refractory to eliciting a transcriptional response following oncogene activation. This refractoriness to BRAF was not because the MC were post-mitotic, as proliferation of the $3 \mathrm{xKO} \pm$ dox $\mathrm{MC}$ was comparable to the proliferation of $3 \mathrm{xKO} \mathrm{NC}$ cells (see Fig. 5G). Because pERK ultimately acts to promote tumorigenesis via activation of downstream transcriptional responses, it appears that the failure of $\mathrm{MC}$ to be transformed is likely related to a failure of BRAF-dependent transcriptional induction in this lineage. This raised the question of what was intrinsically different between these cell types. To address this, we performed RNAseq on WT NC, WT MB and WT MC (i.e. no transgenes) and analyzed the differentially expressed genes by GSEA pathway analysis (table S2). Amongst the most enriched pathways 
that separated the $\mathrm{NC} / \mathrm{MB}$ cells from the MC was the GO term Chromatin modification (NES= 1.901, FDR=0.06, Fig. 3A). In examining individual genes which were enriched in these stages, we found a striking enrichment for many chromatin modifier genes, including readers (e.g. ATAD2, CHD9, BAZ1A), writers (e.g. EZH2, PRMT8, HDAC9) and erasers (e.g. JMJD1C, TET1, TET2), all of which were markedly higher in the NC/MB cells compared to the MC (Fig. 3B and $3 \mathrm{C}$ ). This suggested the possibility that the NC/MB cells might have intrinsically higher capability to rewire their chromatin state in response to $\mathrm{BRAF}^{\mathrm{V} 600 \mathrm{E}}$ and render them competent for melanoma initiation.

To identify which of these chromatin factors is likely most important in establishing competence, we analyzed the top 50 epigenetic-related factors that are higher in MB compared to MC (Fig. 3C) and then asked which of these is most commonly amplified or overexpressed in the human melanoma TCGA cohort. This analysis demonstrated that the top gene was ATAD2, an ATPaseand bromodomain-containing protein (29), which was altered in $20.4 \%$ of melanoma patients (Fig. 3D). We further asked if expression of ATAD2 correlated with patient survival and found that patients in the highest $20 \%$ of expression had significantly worse survival compared to the remaining patients (Fig. 3E and fig. S6A). Although there is no available information about ATAD2 in neural crest or melanoma development, these data nominated it as a key factor for promoting oncogenic competence in the NC/MB lineage. 


\section{ATAD2 acts to reshape chromatin around key NC/MB loci}

We wished to determine if ATAD2 was required for establishment of the NC/MB state and subsequent tumorigenesis. We generated a lentivirus that induced ATAD2 expression in the 3xKO MC (Fig. 4A) to a level comparable to what would be found in the NC cells or MB (fig. S4A). While MC without ATAD2 are deeply pigmented with melanin, reflecting their differentiated state, we noted that the 3xKO MC expressing ATAD2 lost their pigmentation (Fig. 4B). This suggested that ATAD2 expression leads to a dedifferentiated state possibly via affecting chromatin accessibility of NC-related genes in MC (30-33) (Fig. 4B). To test this idea, we performed ATAC-seq on 3xKO dox MB, 3xKO dox MC and 3xKO ATAD2 dox MC to assess global changes in chromatin accessibility. While MC had generally more open chromatin compared to $\mathrm{MB}$, the addition of ATAD2 to the MC did not lead to a global increase in open chromatin (fig. S4B and S4C). Instead we found that overexpression of ATAD2 in the MC led to a significant increase in chromatin accessibility specifically at NC-related loci, to a level comparable if not greater than that found in the 3xKO dox MB themselves (Fig. 4C). GSEA analysis of the affected loci in the 3xKO ATAD2 dox MC versus the 3xKO dox MC showed a significant enrichment of NC-related genes (Fig. 4D, 4E). To gain insight into the transcription factors that are binding to these newly opened chromatin regions, we performed HOMER analysis on the 3xKO ATAD2 dox MC versus 3xKO dox MC. Strikingly, this revealed that the top motif enriched by ATAD2 was SOX10 itself (Fig. 4F, 4H), suggesting that ATAD2 was specifically allowing for SOX10 to bind to its target genes. Analogously, we also asked which peaks became closed after expression of ATAD2 and found that these were most highly enriched for the MITF motif (Fig. 4G, 4I), consistent with the idea that ATAD2 was associated with dedifferentiation and a decreased ability of MITF to activate target genes typically associated 
with differentiation. Network analysis of the loci most affected by ATAD2 and that carried the SOX10 motif showed a strong enrichment for pathways associated with neural precursor proliferation and NC migration (Fig. 4J).

\section{ATAD2 is necessary for NC induction}

Because SOX10 is essential for proper NC induction, these data suggested that loss of ATAD2 might impair proper NC formation. To test this, we utilized the hPSC system in which we could differentiate cells into the NC in the presence or absence of sgRNAs targeting ATAD2. We utilized a previously described inducible iCas 9 system $(34,35)$ to trigger the knockout during hPSC differentiation and found significant reduction with 2 different sgRNAs targeting ATAD2 confirmed by both immunofluorescence (fig. S5A and S5B) and Western blot (fig. S5C). We measured the percentage of NC cells derived from the hPSCs in this assay and found a greater than $50 \%$ reduction in $\mathrm{NC}$ formation with the most potent sgRNA (fig. S5D), in agreement with its requirement for activation of the SOX10 program. When taken together with the observation that the 3xKO ATAD2 dox MC become less pigmented, these data are consistent with the notion that ATAD2 facilitates access to an early NC state, in part by increasing expression of SOX10 target genes while decreasing expression of MITF target genes.

\section{ATAD2 forms a complex with SOX10, allowing for expression of NC genes}

We next asked how ATAD2 might facilitate expression of these NC target genes. Previous work has shown that ATAD2 is able to build a protein complex together with MYC and in this way regulate a MYC-dependent signature in different cancer cell lines (36). We hypothesized that 
ATAD2 might be acting in a similar way with SOX10, by directly binding to it and facilitating transcription of its target genes. In support of this idea, we analyzed genes differentially expressed in the ATAD2 $2^{\mathrm{HI}}$ vs. ATAD2 ${ }^{\mathrm{LO}}$ patients from the TCGA cohort (Fig. 5A and fig. S6A). Pathway analysis revealed a strong MYC signature in the ATAD2 ${ }^{\mathrm{HI}}$ patients (Fig. 5A, 5C and fig. S6B), and motif analysis showed enrichment of the SOX motif (Fig. 5B). To more directly test this hypothesis, we performed co-IP experiments. As previously described, we confirmed that ATAD2 forms a complex with MYC in the 3xKO ATAD2 dox MC (Fig. 5E). Importantly, we also found that ATAD2 and SOX10 are indeed bound in the same protein complex by co-IP (Fig. 5D). Based on these findings, we hypothesized that ATAD2 might play a dual role and facilitate the expression of target genes of both MYC and SOX10 transcription factors. To test this hypothesis, we performed qRT-PCR to measure expression of representative gene targets of both of these transcription factors in the presence or absence of ATAD2 and found a significant increase in expression of key genes such as CD271, ETS1, DDX21 and E2F1. Interestingly, we also noted increases in MYC and SOX10 itself, suggesting a possible feed forward mechanism (Fig. 5F). These data indicate that ATAD2 is a critical factor that enables oncogenic gene programs by interacting with both MYC as well as the NC lineage factor SOX10.

\section{ATAD2 promotes melanoma phenotypes}

Because SOX10 has previously been shown to be required for melanoma initiation, and its function is facilitated via ATAD2, we next wanted to test its role in melanoma initiation and progression. We first assessed in vitro cellular phenotypes using the hPSC-based system. We expressed ATAD2 in the 3xKO MC to a level similar to endogenous expression in MB cells (fig. S4A) and we analyzed their proliferation rates by incorporation of EdU followed by FACS 
analysis (Fig. 5G). We observed that 3xKO ATAD2 \pm dox MC had a comparable proliferation rate to $3 \mathrm{xKO}$ dox $\mathrm{MB}$ and that they were significantly more proliferative than $3 \mathrm{xKO}$ dox $\mathrm{MC}$. Both proliferation rates in $3 \times \mathrm{KO} N C$ cells and $\mathrm{MB}$ increased upon $\mathrm{BRAF}^{\mathrm{V} 600 \mathrm{E}}$ expression, while in the already highly proliferative 3xKO ATAD2 dox MC did not. Noteworthy, in vitro 3xKO $\mathrm{MC}$ were not entering a senescent state upon $\mathrm{BRAF}^{\mathrm{V} 600 \mathrm{E}}$ expression and their proliferation rate did not change. The increased proliferation in $3 x \mathrm{KO}$ ATAD2 \pm dox MC was accompanied by an increase in invasion, as measured by the invasion chamber analysis (fig. S7A, S7B) and as supported by ATAC-seq gene signatures consistent with an epithelial-to-mesenchymal (EMT) program (fig. S7C, S7D). We also assessed the metabolic profile of these hPSC-derived tumor lines and found evidence of significant metabolic rewiring. We used Seahorse assays to measure mitochondrial bioenergetics and glycolysis via oxygen consumption rate (OCR) and the extracellular acidification rate (ECAR). The ratio between OCR and ECAR showed that 3xKO MB were mostly relying on glycolysis for energy production and that this trend was amplified by dox-induced oncogene expression (37-39). On the contrary, 3xKO MC displayed a profoundly different metabolic profile, with sustained oxidative metabolism. Strikingly, upon ATAD2 expression, the $3 \mathrm{xKO}$ ATAD2 dox MC had a reduced oxygen consumption and instead switched to a more glycolytic state, as evidenced by an increased ECAR/OCR ratio (Fig. 5H and fig. S8). These phenotypes all support the notion that overexpression of ATAD2 triggers mechanisms that promote tumorigenic phenotypes.

\section{ATAD2 is required for melanoma initiation in vivo}

Given these in vitro phenotypes and the known reliance on SOX10 for melanoma growth, these data led us to ask whether loss of ATAD2 would prevent melanoma initiation. To do this, we 
established a highly sensitive assay to image and quantify true tumor initiation, rather than monitoring the later steps of tumor progression. We recently developed a method called TEAZ (40) that allows us to initiate melanoma in the zebrafish skin via transgene electroporation. These transgenes can be activated in our previously developed transparent casper strain of zebrafish (41), such that we can directly visualize even a single cell as it becomes transformed by $\mathrm{BRAF}^{\mathrm{V} 600 \mathrm{E}}$. Into a focal region of the dorsal skin of the zebrafish, we electroporated plasmids that will activate mitfa along with $\mathrm{BRAF}^{\mathrm{V} 600 \mathrm{E}}$ and a GFP marker. These were co-electroporated with recombinant Cas9 as well as either non-targeting (NT) sgRNAs or sgRNAs against the zebrafish ortholog of ATAD2 (Fig. 6A). The fish were then monitored from days 3 through 21 post-electroporation, and each fish imaged using brightfield (BF) and GFP fluorescence. In the non-targeting controls, $65 \%$ of the fish developed a patch of $\mathrm{GFP}^{+}$melanocytes (Fig. 6B, 6C and 6D), easily discernible from the surrounding normal skin. In contrast, in the animals which were electroporated with sgRNAs against ATAD2, we found that most fish were negative for any GFP (Fig. 6B, 6C and 6E). Quantification of the $\mathrm{GFP}^{+}$area on day 14 revealed a significant decrease in overall tumor size in the ATAD2 knockout compared with the control fish (Fig. 6B). Taken together, our data supports a model in which high levels of ATAD2 expression, which is found in NC/MB cells, supports the ability of BRAF to initiate tumors by its ability to enhance lineagespecific programs in the cell of origin.

\section{Discussion}

In this study, we have established a new hPSC-derived melanoma model in concert with engineered zebrafish transgenics to investigate oncogenic competence. We showed that the ability of a cell to respond to $\mathrm{BRAF}^{\mathrm{V} 600 \mathrm{E}}$ depends upon the pre-existing transcriptional state of 
that cell, which is in turn dependent upon developmental state at the time of transformation. We find that both $\mathrm{NC}$ and $\mathrm{MB}$ stages are able to respond to $\mathrm{BRAF}^{\mathrm{V} 600 \mathrm{E}}$, and that ATAD2 is an oncogenic competence factor required for melanoma initiation.

$\mathrm{NC}$ and $\mathrm{MB}$ cells are most susceptible to $\mathrm{BRAF}^{\mathrm{V} 600 \mathrm{E}}$ and have intrinsically higher level expression of epigenetic modifiers, which include a diverse array of chromatin readers, writers and erasers. We hypothesized that this might impart these cells with a greater capability to transcriptionally respond to oncogenic insult. Consistent with this, our RNA-seq analysis demonstrated a robust response to $\mathrm{BRAF}^{\mathrm{V} 600 \mathrm{E}}$ only in $\mathrm{NC}$ and $\mathrm{MB}$ cells, with barely any response in the MC. This cannot be wholly explained by oncogene-induced senescence, since we find that the 3xKO MC are proliferative both before and after BRAF induction in vitro (Fig. 5G). Amongst the chromatin modifying enzymes we identified in our RNA-seq analysis, we identified ATAD2 as one example of a protein that allows for proper $\mathrm{BRAF}^{\mathrm{V} 600 \mathrm{E}}$-induced transformation and is amongst the most dysregulated chromatin modifying enzymes in human melanoma.

In normal physiology, ATAD2 is expressed during development in embryonic stem cells, but in adults ATAD2 expression is restricted to the male germ cells and to the bone marrow (42). How ATAD2 acts to affect transcription, and cancer more broadly, remains under investigation. It contains both AAA-ATPase and bromodomains and can bind to acetylated histones $(43,44)$. It has previously been shown to act as a co-regulator of oncogenic transcription factors such as MYC (36), and it has been identified as portending a worse prognosis in a variety of cancers (29, 45-48). One potential mechanism for its action may be via transcription elongation of target genes. The yeast ortholog, Yta7, localizes to the ORFs of highly transcribed genes (49) and may play a role in eviction or degradation of $\mathrm{H} 3$ during the elongation phase of transcription (50) as well as regulate the transcription of histones themselves (51). Our data is consistent with a model 
in which ATAD2 binds to the key NC factor SOX10 and allows for expression of its target genes.

One important difference between our observations and data using genetically engineered mouse models is that mature melanocytes (both zebrafish and hPSC-derived) are relatively resistant to oncogenesis. The most commonly available mouse models of melanoma utilize a Tyrosinase-Cre driver to activate $\mathrm{BRAF}^{\mathrm{V} 600 \mathrm{E}}$ (52-55). These animals can develop melanoma, although this can be accelerated by inactivation of tumor suppressors such as CDKN2A, TP53 or PTEN (56-59). Which cells within these mice act as the melanoma cell of origin has not been fully resolved (1315), but our studies are not precisely comparable to the mouse studies since our zebrafish use Tyrp1-driven $\mathrm{BRAF}^{\mathrm{V} 600 \mathrm{E}}$. One possible explanation for this discrepancy is that in our system the Tyrpl promoter is actually driving expression in a somewhat more fully differentiated melanocyte compared to the Tyr promoter used in mouse studies. Another explanation is that these differences may reflect different biological thresholds for tumorigenesis, in that a different number of DNA lesions may be required to transform melanocytes in human versus mice versus zebrafish. We noted that in our hPSC-derived MC, surprisingly, even with triple knockout (of $\mathrm{RB} 1, \mathrm{TP} 53$, and P16), $\mathrm{BRAF}^{\mathrm{V} 600 \mathrm{E}}$ was still able to induce senescence in vivo. Therefore, in this particular human context these alterations are not enough to overcome oncogene-induced senescence. One possible mechanism affecting different thresholds for oncogenic competence might be the particular microenvironment, which our studies did not explicitly address. It would be important in future studies to ask how oncogenic competence might differ in each potential cell of origin depending on the local microenvironment.

Our data argue for a model in which there may not be a discrete cell of origin of melanoma, but instead support the idea that multiple cells along the spectrum from NC to MC may be capable of 
giving rise to tumors in the right context (Fig. 6F). It suggests that oncogenic competence is related to three interrelated factors that cooperate to determine susceptibility: DNA mutations (e.g. BRAF), cell-type specific transcription factors (e.g. SOX10), and the intrinsic levels of chromatin modifying enzymes which allow for a permissive chromatin landscape (e.g. ATAD2). 


\section{References:}

1. (2020). "Pan-cancer analysis of whole genomes." Nature 578(7793): 82-93.

2. Sack, L. M., T. Davoli, M. Z. Li, Y. Li, Q. Xu, K. Naxerova, E. C. Wooten, R. J. Bernardi, T. D. Martin, T. Chen, Y. Leng, A. C. Liang, K. A. Scorsone, T. F. Westbrook, K. K. Wong and S. and Aneuploidy Patterns." Cell 173(2): 499-514.e423.

3. Haigis, K. M., K. Cichowski and S. J. Elledge (2019). "Tissue-specificity in cancer: The rule, not the exception." Science 363(6432): 1150-1151.

4. White, R. M., J. Cech, S. Ratanasirintrawoot, C. Y. Lin, P. B. Rahl, C. J. Burke, E. Langdon, S. Granter, R. A. Young, S. Morrison, G. N. Wheeler and L. I. Zon (2011). "DHODH modulates transcriptional elongation in the neural crest and melanoma." Nature 471(7339): 518-522.

5. Rambow, F., A. Rogiers, O. Marin-Bejar, S. Aibar, J. Femel, M. Dewaele, P. Karras, D. Brown, Y. H. Chang, M. Debiec-Rychter, C. Adriaens, E. Radaelli, P. Wolter, O. Bechter, R. Dummer, Aerts, A. W. Lund and J. C. Marine (2018). "Toward Minimal Residual Disease-Directed Therapy in Melanoma." Cell 174(4): 843-855.e819.

6. Kaufman, C. K., C. Mosimann, Z. P. Fan, S. Yang, A. J. Thomas, J. Ablain, J. L. Tan, R. D. Fogley, E. van Rooijen, E. J. Hagedorn, C. Ciarlo, R. M. White, D. A. Matos, A. C. Puller, C. emergence of neural crest identity during melanoma initiation." Science 351(6272): aad2197. 
7. McConnell, A. M., J. K. Mito, J. Ablain, M. Dang, L. Formichella, D. E. Fisher and L. I. Zon (2019). "Neural crest state activation in NRAS driven melanoma, but not in NRAS-driven melanocyte expansion." Dev Biol 449(2): 107-114.

8. Shakhova, O., D. Zingg, S. M. Schaefer, L. Hari, G. Civenni, J. Blunschi, S. Claudinot, M. Cinelli and L. Sommer (2012). "Sox10 promotes the formation and maintenance of giant congenital naevi and melanoma." Nat Cell Biol 14(8): 882-890.

9. Heppt, M. V., J. X. Wang, D. M. Hristova, Z. Wei, L. Li, B. Evans, M. Beqiri, S. Zaman, J. Zhang, M. Irmler, C. Berking, R. Besch, J. Beckers, F. J. Rauscher, 3rd, R. A. Sturm, D. E. Fisher, Promotes Melanoma Progression." J Invest Dermatol 138(1): 141-149.

10. Varum, S., A. Baggiolini, L. Zurkirchen, Z. K. Atak, C. Cantu, E. Marzorati, R. Bossart, J. Wouters, J. Hausel, E. Tuncer, D. Zingg, D. Veen, N. John, M. Balz, M. P. Levesque, K. Basler, S. Aerts, N. Zamboni, R. Dummer and L. Sommer (2019). "Yin Yang 1 Orchestrates a Metabolic 24(4): 637-653.e639.

11. Polsky, D. and C. Cordon-Cardo (2003). "Oncogenes in melanoma." Oncogene 22(20): 30873091.

12. Kunz, M. (2014). "Oncogenes in melanoma: an update." Eur J Cell Biol 93(1-2): 1-10. 
Cutaneous Melanoma Induced by Mutant BRaf Arises from Expansion and Dedifferentiation of Mature Pigmented Melanocytes." Cell Stem Cell 21(5): 679-693.e676.

14. Moon, H., L. R. Donahue, E. Choi, P. O. Scumpia, W. E. Lowry, J. K. Grenier, J. Zhu and A. C. White (2017). "Melanocyte Stem Cell Activation and Translocation Initiate Cutaneous Melanoma in Response to UV Exposure." Cell Stem Cell 21(5): 665-678.e666.

15. Sun, Q., W. Lee, Y. Mohri, M. Takeo, C. H. Lim, X. Xu, P. Myung, R. P. Atit, M. M. Taketo, R. S. Moubarak, M. Schober, I. Osman, D. L. Gay, D. Saur, E. K. Nishimura and M. Ito (2019). "A novel mouse model demonstrates that oncogenic melanocyte stem cells engender melanoma resembling human disease." Nat Commun 10(1): 5023. some avian neural crest cells." Nature 335(6186): 161-164.

17. Baggiolini, A., S. Varum, J. M. Mateos, D. Bettosini, N. John, M. Bonalli, U. Ziegler, L. Dimou, H. Clevers, R. Furrer and L. Sommer (2015). "Premigratory and migratory neural crest cells are multipotent in vivo." Cell Stem Cell 16(3): 314-322. Akkuratova, Y. Yang, M. Haring, V. Dyachuk, C. Bock, M. Farlik, M. L. Piacentino, F. Boismoreau, M. M. Hilscher, C. Yokota, X. Qian, M. Nilsson, M. E. Bronner, L. Croci, W. Y. Hsiao, D. A. Guertin, J. F. Brunet, G. G. Consalez, P. Ernfors, K. Fried, P. V. Kharchenko and I. Adameyko (2019). "Spatiotemporal structure of cell fate decisions in murine neural crest." Science 364(6444). 
19. Reed, R. J. and D. D. Leonard (1979). "Neurotropic melanoma. A variant of desmoplastic melanoma." Am J Surg Pathol 3(4): 301-311.

20. Su, A., S. M. Dry, S. W. Binder, J. Said, P. Shintaku and G. P. Sarantopoulos (2014). "Malignant melanoma with neural differentiation: an exceptional case report and brief review of the pertinent literature." Am J Dermatopathol 36(1): e5-9.

21. Agnarsdottir, M., L. Sooman, A. Bolander, S. Stromberg, E. Rexhepaj, M. Bergqvist, F. Ponten, W. Gallagher, J. Lennartsson, S. Ekman, M. Uhlen and H. Hedstrand (2010). "SOX10 expression in superficial spreading and nodular malignant melanomas." Melanoma Res 20(6): 468478. Buckley, A. Ortega and R. C. Seeger (2006). "Prognostic significance of gene expression profiles of metastatic neuroblastomas lacking MYCN gene amplification." J Natl Cancer Inst 98(17): 11931203.

23. Bosenberg, M., V. Muthusamy, D. P. Curley, Z. Wang, C. Hobbs, B. Nelson, C. Nogueira, J. W. Horner, 2nd, R. Depinho and L. Chin (2006). "Characterization of melanocyte-specific inducible Cre recombinase transgenic mice." Genesis 44(5): 262-267.

24. Mica, Y., G. Lee, S. M. Chambers, M. J. Tomishima and L. Studer (2013). "Modeling neural crest induction, melanocyte specification, and disease-related pigmentation defects in hESCs and patient-specific iPSCs." Cell Rep 3(4): 1140-1152. Human Pluripotent Stem Cells." J Vis Exp(109): e53806. 
26. Quintana, E., M. Shackleton, H. R. Foster, D. R. Fullen, M. S. Sabel, T. M. Johnson and S. J. Morrison (2010). "Phenotypic heterogeneity among tumorigenic melanoma cells from patients that is reversible and not hierarchically organized." Cancer Cell 18(5): 510-523.

27. Quintana, E., M. Shackleton, M. S. Sabel, D. R. Fullen, T. M. Johnson and S. J. Morrison (2008). "Efficient tumour formation by single human melanoma cells." Nature 456(7222): 593598.

28. Tsoi, J., L. Robert, K. Paraiso, C. Galvan, K. M. Sheu, J. Lay, D. J. L. Wong, M. Atefi, R. Shirazi, X. Wang, D. Braas, C. S. Grasso, N. Palaskas, A. Ribas and T. G. Graeber (2018). "Multistage Differentiation Defines Melanoma Subtypes with Differential Vulnerability to Drug-Induced Iron-Dependent Oxidative Stress." Cancer Cell 33(5): 890-904.e895.

29. Boussouar, F., M. Jamshidikia, Y. Morozumi, S. Rousseaux and S. Khochbin (2013). "Malignant genome reprogramming by ATAD2." Biochim Biophys Acta 1829(10): 1010-1014.

30. Hoek, K. S., O. M. Eichhoff, N. C. Schlegel, U. Dobbeling, N. Kobert, L. Schaerer, S. Hemmi and R. Dummer (2008). "In vivo switching of human melanoma cells between proliferative and invasive states." Cancer Res 68(3): 650-656.

31. Landsberg, J., J. Kohlmeyer, M. Renn, T. Bald, M. Rogava, M. Cron, M. Fatho, V. Lennerz, T. Wolfel, M. Holzel and T. Tuting (2012). "Melanomas resist T-cell therapy through inflammation-induced reversible dedifferentiation." Nature 490(7420): 412-416.

32. Konieczkowski, D. J., C. M. Johannessen, O. Abudayyeh, J. W. Kim, Z. A. Cooper, A. Piris, D. T. Frederick, M. Barzily-Rokni, R. Straussman, R. Haq, D. E. Fisher, J. P. Mesirov, W. C. 
Hahn, K. T. Flaherty, J. A. Wargo, P. Tamayo and L. A. Garraway (2014). "A melanoma cell state distinction influences sensitivity to MAPK pathway inhibitors." Cancer Discov 4(7): 816-827.

33. Muller, J., O. Krijgsman, J. Tsoi, L. Robert, W. Hugo, C. Song, X. Kong, P. A. Possik, P. D. Cornelissen-Steijger, M. H. Geukes Foppen, K. Kemper, C. R. Goding, U. McDermott, C. Blank,

J. Haanen, T. G. Graeber, A. Ribas, R. S. Lo and D. S. Peeper (2014). "Low MITF/AXL ratio predicts early resistance to multiple targeted drugs in melanoma." Nat Commun 5: 5712.

34. Gonzalez, F., Z. Zhu, Z. D. Shi, K. Lelli, N. Verma, Q. V. Li and D. Huangfu (2014). "An iCRISPR platform for rapid, multiplexable, and inducible genome editing in human pluripotent stem cells." Cell Stem Cell 15(2): 215-226.

35. Sanjana, N. E., O. Shalem and F. Zhang (2014). "Improved vectors and genome-wide libraries for CRISPR screening." Nat Methods 11(8): 783-784.

36. Ciro, M., E. Prosperini, M. Quarto, U. Grazini, J. Walfridsson, F. McBlane, P. Nucifero, G. Pacchiana, M. Capra, J. Christensen and K. Helin (2009). "ATAD2 is a novel cofactor for MYC, overexpressed and amplified in aggressive tumors." Cancer Res 69(21): 8491-8498.

Thorup, S. M. Moghimi, P. B. Jensen, J. Bartek, P. Guldberg and C. Christensen (2013). "Dysfunctional oxidative phosphorylation makes malignant melanoma cells addicted to glycolysis driven by the (V600E)BRAF oncogene." Oncotarget 4(4): 584-599.

38. Parmenter, T. J., M. Kleinschmidt, K. M. Kinross, S. T. Bond, J. Li, M. R. Kaadige, A. Rao,

K. E. Sheppard, W. Hugo, G. M. Pupo, R. B. Pearson, S. L. McGee, G. V. Long, R. A. Scolyer, H. Rizos, R. S. Lo, C. Cullinane, D. E. Ayer, A. Ribas, R. W. Johnstone, R. J. Hicks and G. A. 
McArthur (2014). "Response of BRAF-mutant melanoma to BRAF inhibition is mediated by a network of transcriptional regulators of glycolysis." Cancer Discov 4(4): 423-433.

39. Nagarajan, A., P. Malvi and N. Wajapeyee (2016). "Oncogene-directed alterations in cancer cell metabolism." Trends Cancer 2(7): 365-377.

40. Callahan, S. J., S. Tepan, Y. M. Zhang, H. Lindsay, A. Burger, N. R. Campbell, I. S. Kim, T. J. Hollmann, L. Studer, C. Mosimann and R. M. White (2018). "Cancer modeling by Transgene Electroporation in Adult Zebrafish (TEAZ)." Dis Model Mech 11(9).

41. White, R. M., A. Sessa, C. Burke, T. Bowman, J. LeBlanc, C. Ceol, C. Bourque, M. Dovey, W. Goessling, C. E. Burns and L. I. Zon (2008). "Transparent adult zebrafish as a tool for in vivo transplantation analysis." Cell Stem Cell 2(2): 183-189.

42. Morozumi, Y., F. Boussouar, M. Tan, A. Chaikuad, M. Jamshidikia, G. Colak, H. He, L. Nie, C. Petosa, M. de Dieuleveult, S. Curtet, A. L. Vitte, C. Rabatel, A. Debernardi, F. L. Cosset, E. Verhoeyen, A. Emadali, N. Schweifer, D. Gianni, M. Gut, P. Guardiola, S. Rousseaux, M. Gerard, S. Knapp, Y. Zhao and S. Khochbin (2016). "Atad2 is a generalist facilitator of chromatin dynamics in embryonic stem cells." J Mol Cell Biol 8(4): 349-362.

43. Koo, S. J., A. E. Fernandez-Montalvan, V. Badock, C. J. Ott, S. J. Holton, O. von Ahsen, J. Toedling, S. Vittori, J. E. Bradner and M. Gorjanacz (2016). "ATAD2 is an epigenetic reader of newly synthesized histone marks during DNA replication." Oncotarget 7(43): 70323-70335.

44. Langini, C., A. Caflisch and A. Vitalis (2017). "The ATAD2 bromodomain binds different acetylation marks on the histone H4 in similar fuzzy complexes." J Biol Chem 292(40): 1673416745. 
45. Zou, J. X., A. S. Revenko, L. B. Li, A. T. Gemo and H. W. Chen (2007). "ANCCA, an estrogenregulated AAA + ATPase coactivator for ERalpha, is required for coregulator occupancy and chromatin modification." Proc Natl Acad Sci U S A 104(46): 18067-18072.

46. Revenko, A. S., E. V. Kalashnikova, A. T. Gemo, J. X. Zou and H. W. Chen (2010). "Chromatin loading of E2F-MLL complex by cancer-associated coregulator ANCCA via reading a specific histone mark." Mol Cell Biol 30(22): 5260-5272.

47. Zou, J. X., L. Guo, A. S. Revenko, C. G. Tepper, A. T. Gemo, H. J. Kung and H. W. Chen (2009). "Androgen-induced coactivator ANCCA mediates specific androgen receptor signaling in prostate cancer." Cancer Res 69(8): 3339-3346. C. Brambilla, E. Brambilla, S. Rousseaux and S. Khochbin (2010). "Functional characterization of ATAD2 as a new cancer/testis factor and a predictor of poor prognosis in breast and lung cancers." Oncogene 29(37): 5171-5181.

49. Lombardi, L. M., A. Ellahi and J. Rine (2011). "Direct regulation of nucleosome density by the conserved AAA-ATPase Yta7." Proc Natl Acad Sci U S A 108(49): E1302-1311.

50. Cattaneo, M., Y. Morozumi, D. Perazza, F. Boussouar, M. Jamshidikia, S. Rousseaux, A. Verdel and S. Khochbin (2014). "Lessons from yeast on emerging roles of the ATAD2 protein family in gene regulation and genome organization." Mol Cells 37(12): 851-856.

51. Kurat, C. F., J. P. Lambert, D. van Dyk, K. Tsui, H. van Bakel, S. Kaluarachchi, H. Friesen, P. 
transcription to S phase by phosphorylation of a chromatin boundary protein." Genes Dev 25(23): 2489-2501.

52. Yajima, I., E. Belloir, Y. Bourgeois, M. Kumasaka, V. Delmas and L. Larue (2006). "Spatiotemporal gene control by the Cre-ERT2 system in melanocytes." Genesis 44(1): 34-43.

53. Dhomen, N., J. S. Reis-Filho, S. da Rocha Dias, R. Hayward, K. Savage, V. Delmas, L. Larue, C. Pritchard and R. Marais (2009). "Oncogenic Braf induces melanocyte senescence and melanoma in mice." Cancer Cell 15(4): 294-303.

54. Goel, V. K., N. Ibrahim, G. Jiang, M. Singhal, S. Fee, T. Flotte, S. Westmoreland, F. S. Haluska, P. W. Hinds and F. G. Haluska (2009). "Melanocytic nevus-like hyperplasia and melanoma in transgenic BRAFV600E mice." Oncogene 28(23): 2289-2298.

55. Marsh Durban, V., M. M. Deuker, M. W. Bosenberg, W. Phillips and M. McMahon (2013). "Differential AKT dependency displayed by mouse models of BRAFV600E-initiated melanoma." J Clin Invest 123(12): 5104-5118.

56. Dankort, D., D. P. Curley, R. A. Cartlidge, B. Nelson, A. N. Karnezis, W. E. Damsky, Jr., M.

J. You, R. A. DePinho, M. McMahon and M. Bosenberg (2009). "Braf(V600E) cooperates with Pten loss to induce metastatic melanoma." Nat Genet 41(5): 544-552.

57. Viros, A., B. Sanchez-Laorden, M. Pedersen, S. J. Furney, J. Rae, K. Hogan, S. Ejiama, M. R. Girotti, M. Cook, N. Dhomen and R. Marais (2014). "Ultraviolet radiation accelerates BRAFdriven melanomagenesis by targeting TP53." Nature 511(7510): 478-482. 
58. Damsky, W., G. Micevic, K. Meeth, V. Muthusamy, D. P. Curley, M. Santhanakrishnan, I. Erdelyi, J. T. Platt, L. Huang, N. Theodosakis, M. R. Zaidi, S. Tighe, M. A. Davies, D. Dankort, M. McMahon, G. Merlino, N. Bardeesy and M. Bosenberg (2015). "mTORC1 activation blocks BrafV600E-induced growth arrest but is insufficient for melanoma formation." Cancer Cell 27(1): 41-56.

59. Perez-Guijarro, E., C. P. Day, G. Merlino and M. R. Zaidi (2017). "Genetically engineered mouse models of melanoma." Cancer 123(S11): 2089-2103.

60. Berghmans, S., R. D. Murphey, E. Wienholds, D. Neuberg, J. L. Kutok, C. D. Fletcher, J. P. Morris, T. X. Liu, S. Schulte-Merker, J. P. Kanki, R. Plasterk, L. I. Zon and A. T. Look (2005). "tp53 mutant zebrafish develop malignant peripheral nerve sheath tumors." Proc Natl Acad Sci U S A 102(2): 407-412.

61. Patton, E. E., H. R. Widlund, J. L. Kutok, K. R. Kopani, J. F. Amatruda, R. D. Murphey, S. Berghmans, E. A. Mayhall, D. Traver, C. D. Fletcher, J. C. Aster, S. R. Granter, A. T. Look, C. Lee, D. E. Fisher and L. I. Zon (2005). "BRAF mutations are sufficient to promote nevi formation and cooperate with p53 in the genesis of melanoma." Curr Biol 15(3): 249-254.

62. Ceol, C. J., Y. Houvras, J. Jane-Valbuena, S. Bilodeau, D. A. Orlando, V. Battisti, L. Fritsch, W. M. Lin, T. J. Hollmann, F. Ferre, C. Bourque, C. J. Burke, L. Turner, A. Uong, L. A. Johnson, R. Beroukhim, C. H. Mermel, M. Loda, S. Ait-Si-Ali, L. A. Garraway, R. A. Young and L. I. Zon (2011). "The histone methyltransferase SETDB1 is recurrently amplified in melanoma and accelerates its onset." Nature 471(7339): 513-517. 
63. D'Agati, G., R. Beltre, A. Sessa, A. Burger, Y. Zhou, C. Mosimann and R. M. White (2017). "A defect in the mitochondrial protein Mpv17 underlies the transparent casper zebrafish." Dev Biol 430(1): 11-17.

64. Perez, A. R., Y. Pritykin, J. A. Vidigal, S. Chhangawala, L. Zamparo, C. S. Leslie and A. Ventura (2017). "GuideScan software for improved single and paired CRISPR guide RNA design." Nat Biotechnol 35(4): 347-349.

65. Labun, K., T. G. Montague, M. Krause, Y. N. Torres Cleuren, H. Tjeldnes and E. Valen (2019). "CHOPCHOP v3: expanding the CRISPR web toolbox beyond genome editing." Nucleic Acids Res 47(W1): W171-w174. (2009). "Highly efficient neural conversion of human ES and iPS cells by dual inhibition of SMAD signaling." Nat Biotechnol 27(3): 275-280.

67. Lee, G., S. M. Chambers, M. J. Tomishima and L. Studer (2010). "Derivation of neural crest cells from human pluripotent stem cells." Nat Protoc 5(4): 688-701. in human pluripotent stem cells." Methods Enzymol 546: 215-250.

69. Haeussler, M., K. Schonig, H. Eckert, A. Eschstruth, J. Mianne, J. B. Renaud, S. SchneiderMaunoury, A. Shkumatava, L. Teboul, J. Kent, J. S. Joly and J. P. Concordet (2016). "Evaluation of off-target and on-target scoring algorithms and integration into the guide RNA selection tool CRISPOR." Genome Biol 17(1): 148. 
70. Eisenberg, E. and E. Y. Levanon (2013). "Human housekeeping genes, revisited." Trends Genet 29(10): 569-574.

71. Bolger, A. M., M. Lohse and B. Usadel (2014). "Trimmomatic: a flexible trimmer for Illumina sequence data." Bioinformatics 30(15): 2114-2120. and T. R. Gingeras (2013). "STAR: ultrafast universal RNA-seq aligner." Bioinformatics 29(1): 15-21.

73. DeLuca, D. S., J. Z. Levin, A. Sivachenko, T. Fennell, M. D. Nazaire, C. Williams, M. Reich, W. Winckler and G. Getz (2012). "RNA-SeQC: RNA-seq metrics for quality control and process optimization." Bioinformatics 28(11): 1530-1532.

74. Love, M. I., W. Huber and S. Anders (2014). "Moderated estimation of fold change and dispersion for RNA-seq data with DESeq2." Genome Biol 15(12): 550.

75. Xiong, Q., N. Ancona, E. R. Hauser, S. Mukherjee and T. S. Furey (2012). "Integrating genetic and gene expression evidence into genome-wide association analysis of gene sets." Genome Res 22(2): 386-397.

76. Xiong, Q., S. Mukherjee and T. S. Furey (2014). "GSAASeqSP: a toolset for gene set association analysis of RNA-Seq data." Sci Rep 4: 6347.

77. Hu, Y., I. Flockhart, A. Vinayagam, C. Bergwitz, B. Berger, N. Perrimon and S. E. Mohr (2011). "An integrative approach to ortholog prediction for disease-focused and other functional studies." BMC Bioinformatics 12: 357. 
78. Buenrostro, J. D., P. G. Giresi, L. C. Zaba, H. Y. Chang and W. J. Greenleaf (2013). "Transposition of native chromatin for fast and sensitive epigenomic profiling of open chromatin, DNA-binding proteins and nucleosome position." Nat Methods 10(12): 1213-1218. Shmulevich, C. Sander and J. M. Stuart (2013). "The Cancer Genome Atlas Pan-Cancer analysis project." Nat Genet 45(10): 1113-1120.

80. Liu, J., T. Lichtenberg, K. A. Hoadley, L. M. Poisson, A. J. Lazar, A. D. Cherniack, A. J. Kovatich, C. C. Benz, D. A. Levine, A. V. Lee, L. Omberg, D. M. Wolf, C. D. Shriver, V. Thorsson and H. Hu (2018). "An Integrated TCGA Pan-Cancer Clinical Data Resource to Drive HighQuality Survival Outcome Analytics." Cell 173(2): 400-416.e411.

81. Colaprico, A., T. C. Silva, C. Olsen, L. Garofano, C. Cava, D. Garolini, T. S. Sabedot, T. M. Malta, S. M. Pagnotta, I. Castiglioni, M. Ceccarelli, G. Bontempi and H. Noushmehr (2016). "TCGAbiolinks: an R/Bioconductor package for integrative analysis of TCGA data." Nucleic Acids Res 44(8): e71.

82. Silva, T. C., A. Colaprico, C. Olsen, F. D'Angelo, G. Bontempi, M. Ceccarelli and H. Noushmehr (2016). "TCGA Workflow: Analyze cancer genomics and epigenomics data using Bioconductor packages." F1000Res 5: 1542.

83. Risso, D., K. Schwartz, G. Sherlock and S. Dudoit (2011). "GC-content normalization for RNA-Seq data." BMC Bioinformatics 12: 480. 


\section{Acknowledgments:}

We are grateful to the members of the Studer laboratory and of the White laboratory for helpful discussions and support for this project. We are also thankful to the members of the Integrated Genomics Operation Core (MSKCC) for the RNA-sequencing studies, the Epigenetics Core (MSKCC) for the ATAC sequencing, the Flow Cytometry Core (MSKCC) for the cell-sorting applications, the Metabolism Core (MSKCC) for the seahorse applications, the Molecular Cytogenetics Core (MKSCC) for karyotyping and the Antitumor Assessment Core (MSKCC) for some of the xenograft studies.

This work was supported by awards from the Melanoma Research Alliance (R.M.W. and L.S.), predoctoral fellowship F31CA196305, Joanna M. Nicolay Melanoma Foundation Research Scholar Award and the Robert B. Catell Fellowship (all to S.J.C.), Kirschstein-NRSA predoctoral fellowship F30CA220954, Melanoma Research Foundation, Medical Scientist Training Program T32GM007739 (all to N.R.C.), NIH Kirschstein-NRSA Predoctoral

Fellowship F30CA236442, Molecular and Cell Biology Teaching Grant T32GM008539,

Medical Scientist Training Program T32GM007739 (all to J.M.W), the Swiss National Science Foundation Early Postdoc.mobility fellowship P2ZHP3_171967 and the Postdoc.mobility fellowship P400PB_180672 (all to A.B.) and P30 CA008748 (NCI Core Facility Grant). 


\section{Competing interests:}

L.S. is co-founder and consultant of BlueRock Therapeutics and is listed as inventor on patent application by MSKCC related to melanocyte differentiation from human pluripotent stem cells (WO2011149762A2). R.M.W. is a consultant to N-of-One, a subsidiary of Qiagen. All other authors declare no competing interests.

\section{Data and materials availability:}

GEO accession numbers for RNA-seq and ATAC-seq data are pending. All raw data files will be made available upon request. All transgenic zebrafish lines are available upon request from the authors or via the ZIRC zebrafish stock center (https://zebrafish.org/home/guide.php).

\section{Supplementary Materials:}

Materials and Methods

Figures S1-S8 
FigbiqRxiv preprint doi: https://doi.org/10.1101/2020.05.09.081554; this version posted May 10, 2020. The copyright holder for this preprint (which was not certified by peer review) is the author/funder. All rights reserved. No reuse allowed without permission.

A

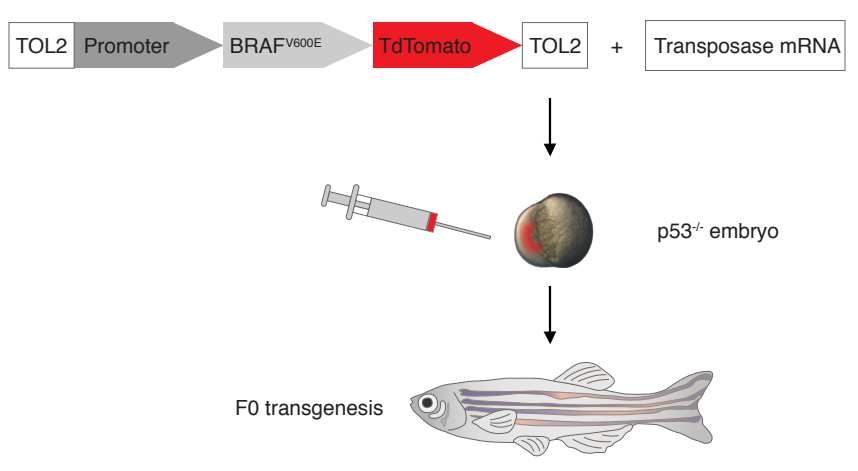

B

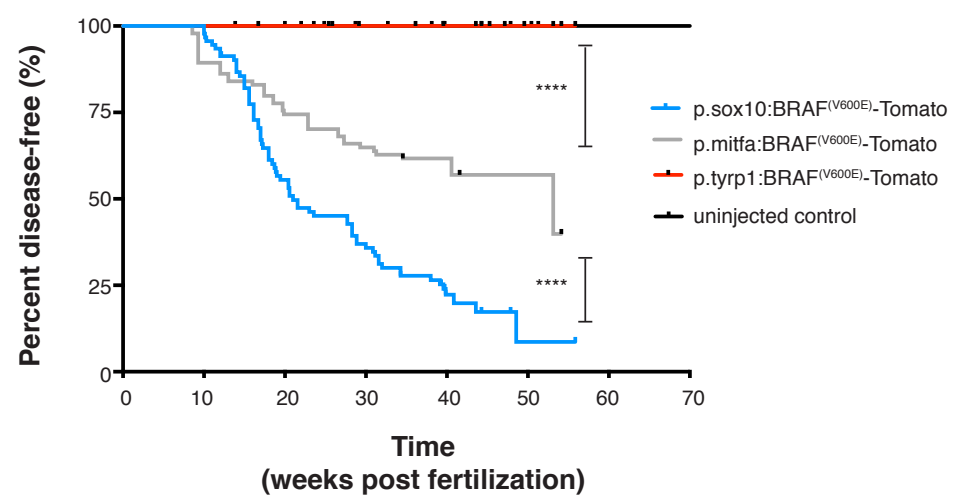

C p.sox10:BRAF( ${ }^{(\mathrm{V} 600 \mathrm{E})}$-Tomato

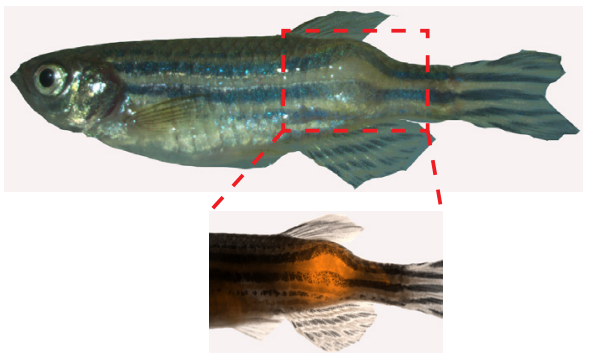

F

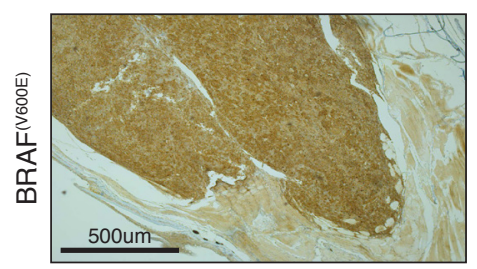

I

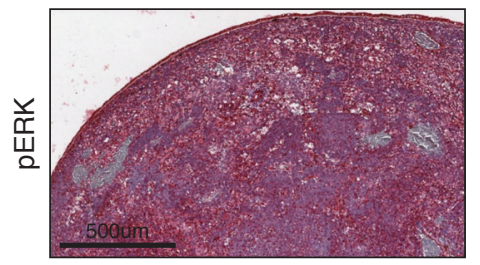

$\mathbf{L}$

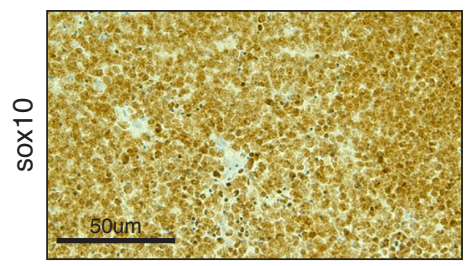

$\mathbf{N}$

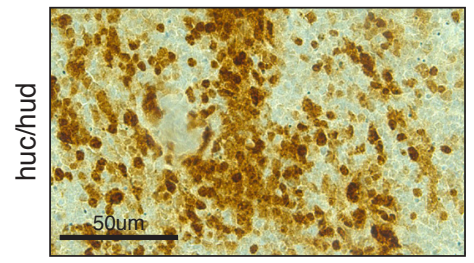

$\mathbf{P}$

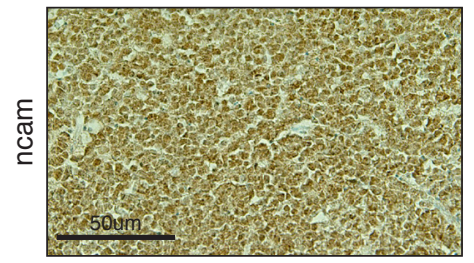

D p.mitfa:BRAF( ${ }^{(\mathrm{V} 600 \mathrm{E})}$-Tomato

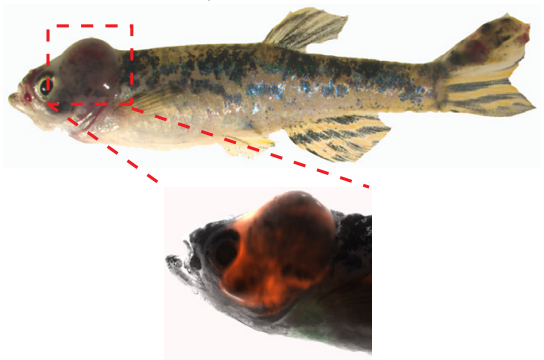

G

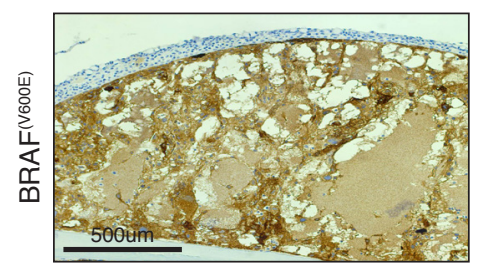

$\mathbf{J}$

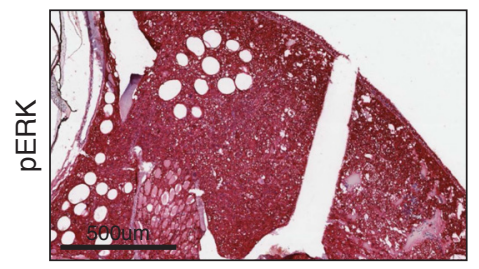

M

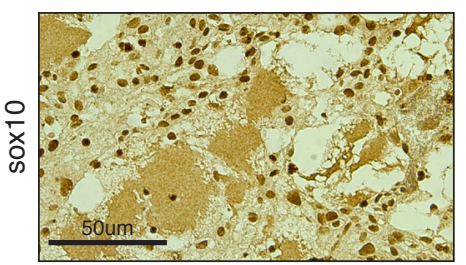

0

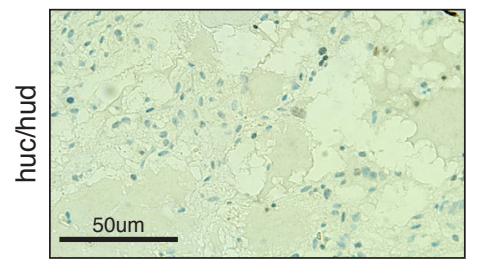

Q

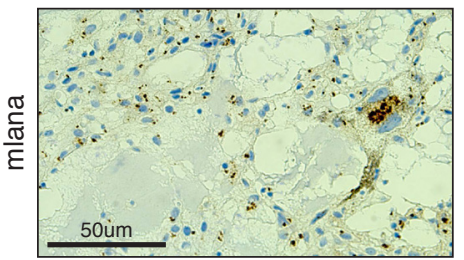

E p.tyrp1:BRAF(v600E)-Tomato

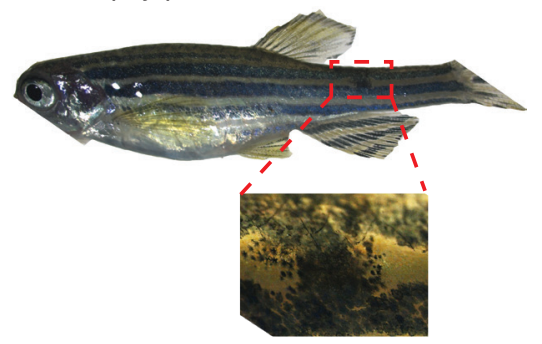

H

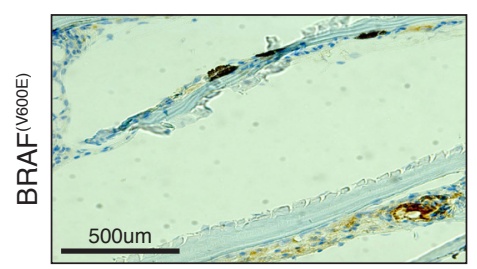

K

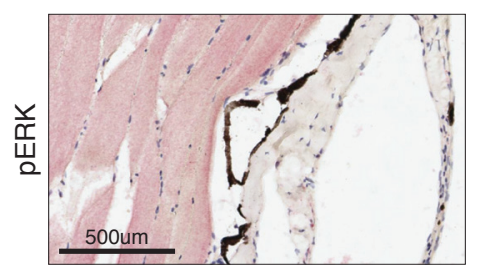

$\mathbf{R}$ p.mitfa:BRAF(v600e)-Tomato

- p.sox10:BRAF( ${ }^{\left({ }^{6} 00 E\right)}$-Tomato

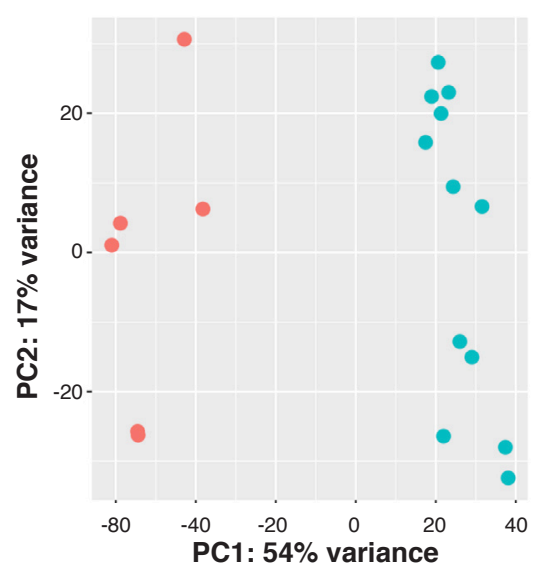




\section{Fig. 1. Zebrafish models show that the NC and MB states, but not the MC state, are}

\section{cancer competent.}

(A) Schematic drawing of zebrafish F0 transgenesis. F0 zebrafish transgenic fish were engineered by injection of $\mathrm{p} 53^{-/-}$single-cell embryos with transposase mRNA together with TOL2 flanked plasmids, which encoded a stage-specific promoter (sox10, mitf, tyrp1) driving BRAF( ${ }^{(\mathrm{V} 600 \mathrm{E})}$ fused to TdTomato.

(B) Kaplan-Meier curves of F0 $\mathrm{p} 53^{-/-}$transgenic zebrafish injected with plasmids driving $\mathrm{BRAF}^{(\mathrm{V} 600 \mathrm{E})}$ fused to TdTomato under either the NC-specific promoter $\operatorname{sox} 10(\mathrm{n}=92$ biological replicates), the MB-specific promoter mitfa ( $\mathrm{n}=94$ biological replicates), or the MC-specific promoter tyrpl ( $\mathrm{n}=49$ biological replicates) or uninfected control $(\mathrm{n}=86$ biological replicates). $* * * *=p<0.0001$ for the comparison of the tumor-free survival curves of fish with MB-derived tumors and MC-derived nevus-like structures; $* * * *=\mathrm{p}<$ 0.0001 for the comparison of the tumor-free survival curves of fish with NC- and MBderived tumors; log-rank (Mantel-Cox) test.

(C) Representative NC-derived tumor developed in the sox 10:BRAF(V600E) $\mathrm{p}^{-1-}$ transgenic fish.

(D) Representative MB-derived tumor developed in the mitfa:BRAF ${ }^{(\mathrm{V} 600 \mathrm{E})}{\mathrm{p} 53^{-/}}^{-}$ transgenic fish.

(E) Representative nevus-like structure developed in the tyrp 1:BRAF ${ }^{(\mathrm{V} 600 \mathrm{E})}{\mathrm{p} 53^{-/-}}^{-1}$ transgenic fish.

$(\mathbf{F}-\mathbf{K})$ Immunohistochemistry for $\mathrm{BRAF}^{(\mathrm{V} 600 \mathrm{E})}$ and phosphoERK in the NC- and MBderived tumors and in the MC-derived nevus-like structure. 
(L-Q) Immunohistochemistry staining for sox 10, huc/hud, ncam and mlana. NC-derived tumors were positive for the neuronal marks huc/hud and ncam (N, P) and mostly negative for sox10 expression (L). MB-derived tumors were melanomas positive for sox10 (M), mlana (Q), and negative for the neuronal marks huc/hud (O).

(R) PCA plot of mitfa-derived tumors (n=6, M, red) and sox 10-derived tumors $(\mathrm{n}=12, \mathrm{~S}$, blue) for whole genome RNA-seq demonstrated a clear separation at the transcriptional level. 
FigbigRxiv preprint doi: https://doi.org/10.1101/2020.05.09.081554; this version posted May 10, 2020. The copyright holder for this preprint (which was not certified by peer review) is the author/funder. All rights reserved. No reuse allowed without permission.

A

\section{and}
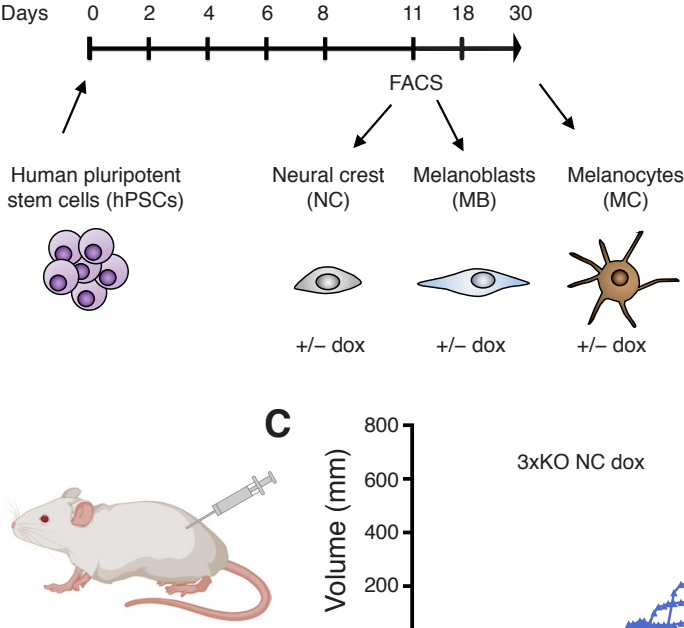

C
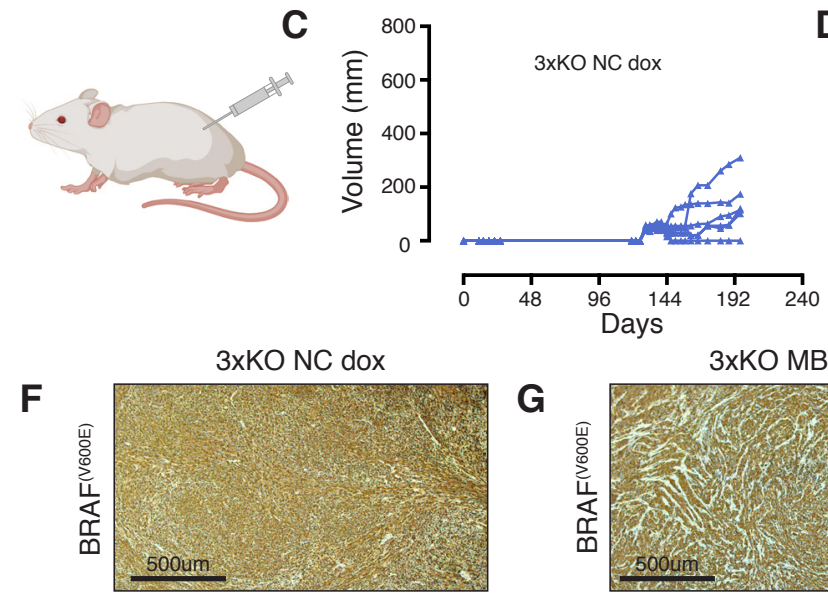

H

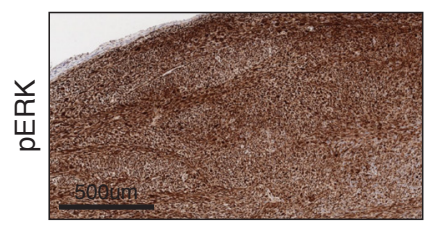

$\mathbf{J}$

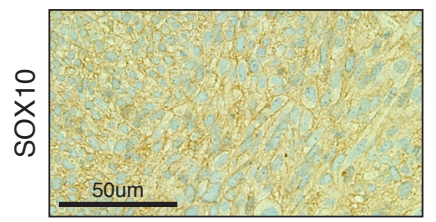

$\mathbf{L}$

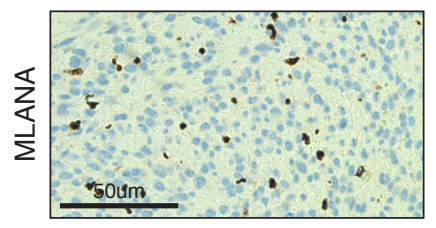

$\mathbf{N}$

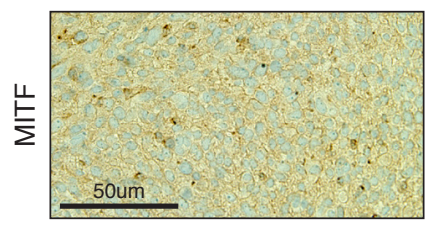

P

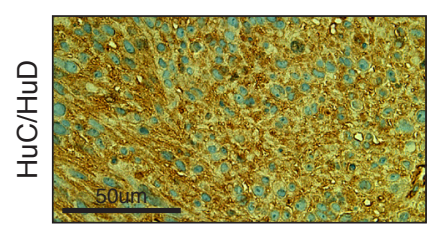

$\mathbf{R}$

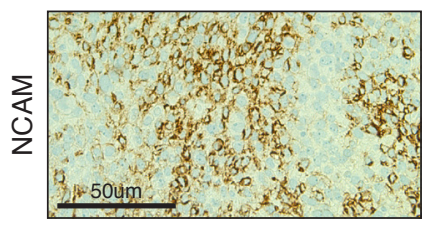

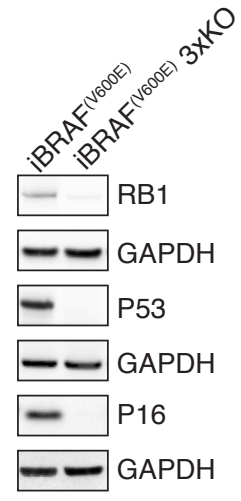

D

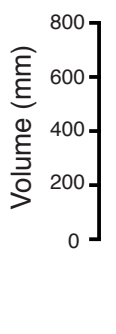

$3 \times K O M B$ dox

G
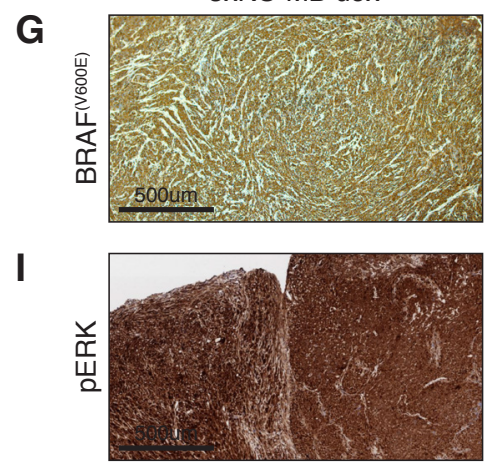

K

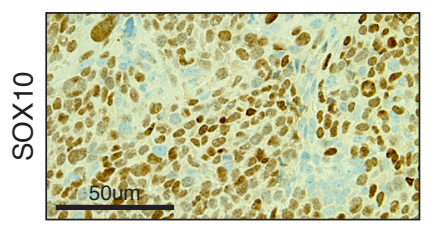

M

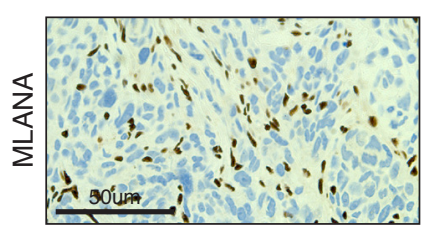

0

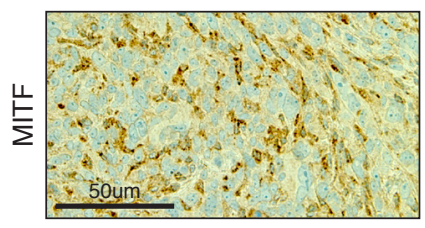

Q

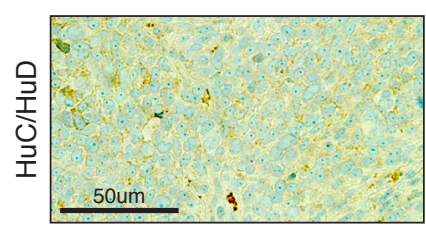

S

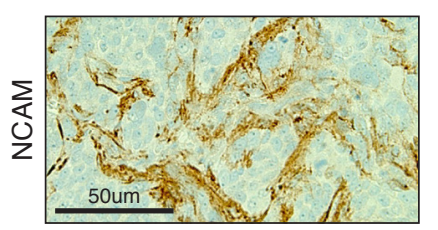

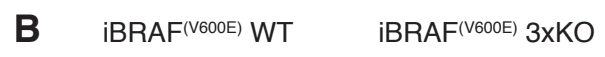

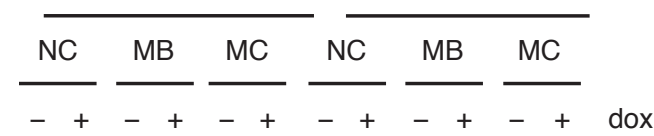

- BRAF(v600)

$\square \square \square 00$ peRK $1 / 2$

$-0-0-0=0-0-0$ totERK $1 / 2$

- - - - $\mathrm{H} 3$

E

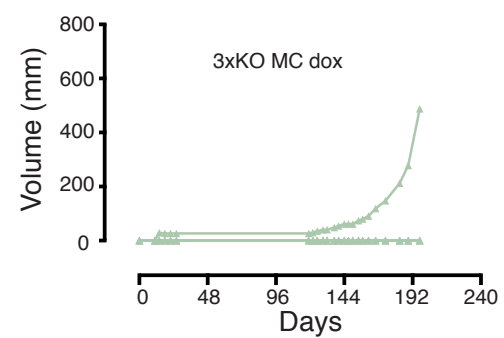

$\mathbf{T}$

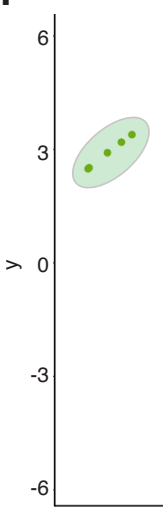

U

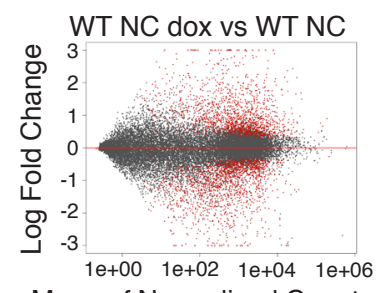

Mean of Normalized Counts

W

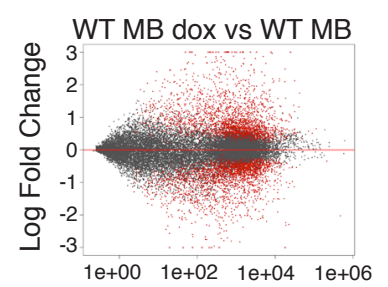

Mean of Normalized Counts

Y

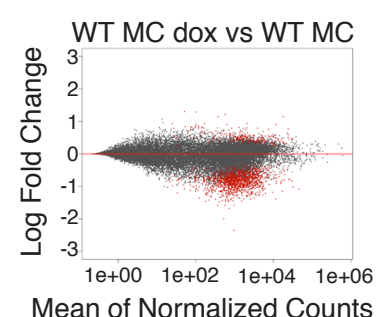

tSNE using Tsoi signature

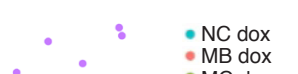

MB dox

- Patient
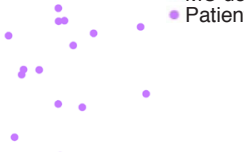

0

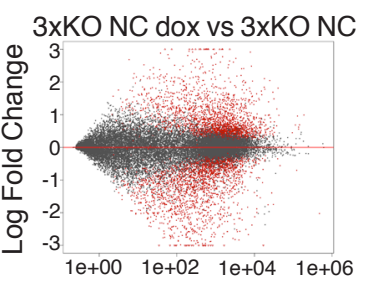

Mean of Normalized Counts

X

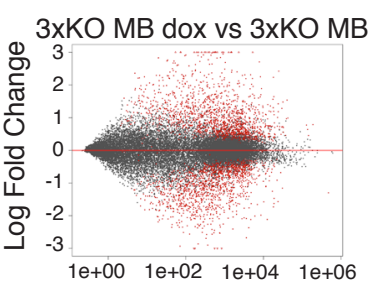

Mean of Normalized Counts

Z 3xKO MC dox vs 3xKO MC

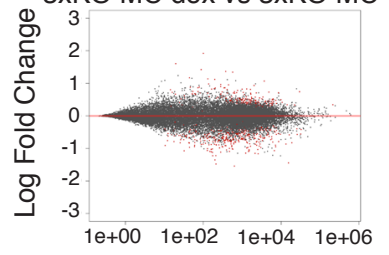

Mean of Normalized Counts 


\section{Fig. 2. A hPSC-based cancer model recapitulates the zebrafish models and}

\section{demonstrates that human NC and MB states are cancer competent, while the differentiated MC state is not.}

(A) Schematic summary of hPSCs differentiation into NC cells, MB and MC and Western blot of the dox-inducible $\mathrm{BRAF}^{(\mathrm{V} 600 \mathrm{E})}\left(\mathrm{iBRAF}^{(\mathrm{V} 600 \mathrm{E})}\right)$ hPSC line knockout for RB1, P53 and P16 (3xKO) using CRISPR/Cas9 technology.

(B) Western blot of $\mathrm{NC}$ cells, $\mathrm{MB}$ and $\mathrm{MC}$ differentiated from either the $\mathrm{BBRAF}^{(\mathrm{V} 600 \mathrm{E})}$

WT or the $\mathrm{iBRAF}^{(\mathrm{V} 600 \mathrm{E})} 3 \mathrm{xKO} \mathrm{hPSCs}$. The cells were exposed to dox $(1 \mu \mathrm{g} / \mathrm{ml})$ for $72 \mathrm{~h}$.

(C-E) In vivo growth curves of 3xKO NC cells + dox ( $n=6$ per group) (C); in vivo growth curves of $3 \mathrm{xKO} \mathrm{MB}+\operatorname{dox}(\mathrm{n}=6$ per group) (D). $3 \mathrm{xKO} \mathrm{MC}+$ dox were not able to grow in vivo ( $\mathrm{n}=6$ per group, 1 outlier) $(\mathbf{E})$, but gave rise to nevus-like structures (Figure S2K). hPSCs-derived cells were injected subcutaneously in immunodeficient NSG mice exposed to a dox-containing diet.

(F-S) Immunohistochemistry of NC-derived and MB-derived tumors + dox treatment.

NC-derived tumors were undifferentiated and heterogeneous tumors, with strong neuronal features $(\mathbf{P}, \mathbf{R})$. MB-derived tumors were diagnosed as melanomas and they were positive for all the common melanocytic marks $(\mathbf{K}, \mathbf{M}, \mathbf{O})$.

(T) t-distributed Stochastic Neighbor Embedding (t-SNE) of 3xKO + dox NC cells, MB and MC samples and the TCGA melanoma samples using the Tsoi signature for melanoma subtypes.

(U-Z) MA plots of the RNA-seq of WT NC cells, MB and MC \pm dox treatment and $3 x K O N C$ cells, $\mathrm{MB}$ and $\mathrm{MC} \pm$ dox treatment ( $\mathrm{n}=3$ per condition). The mean of normalized counts of each gene was plotted against the log fold change following dox- 
bioRxiv preprint doi: https://doi.org/10.1101/2020.05.09.081554; this version posted May 10, 2020. The copyright holder for this preprint (which was not certified by peer review) is the author/funder. All rights reserved. No reuse allowed without permission.

induced $\mathrm{BRAF}^{(\mathrm{V} 600 \mathrm{E})}$ expression within that condition. Adjusted $\mathrm{p}$ value cut-off of 0.05

was used for significantly differentially expressed genes (red). 
Fig:-gixiv preprint doi: https://doi.org/10.1101/2020.05.09.081554; this version posted May 10, 2020. The copyright holder for this preprint (which was not certified by peer review) is the author/funder. All rights reserved. No reuse allowed without permission.

A

GO Term: Chromatin modification

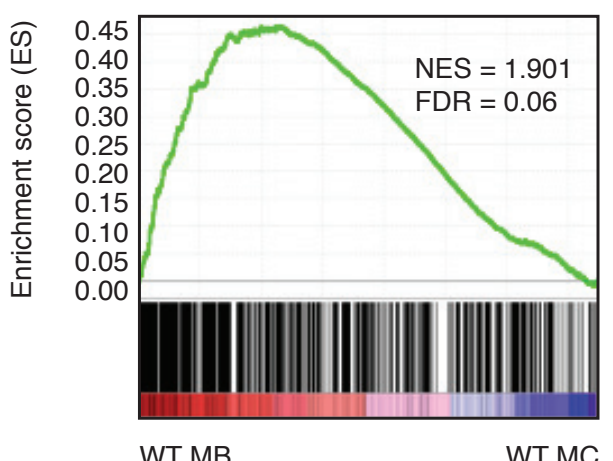

C

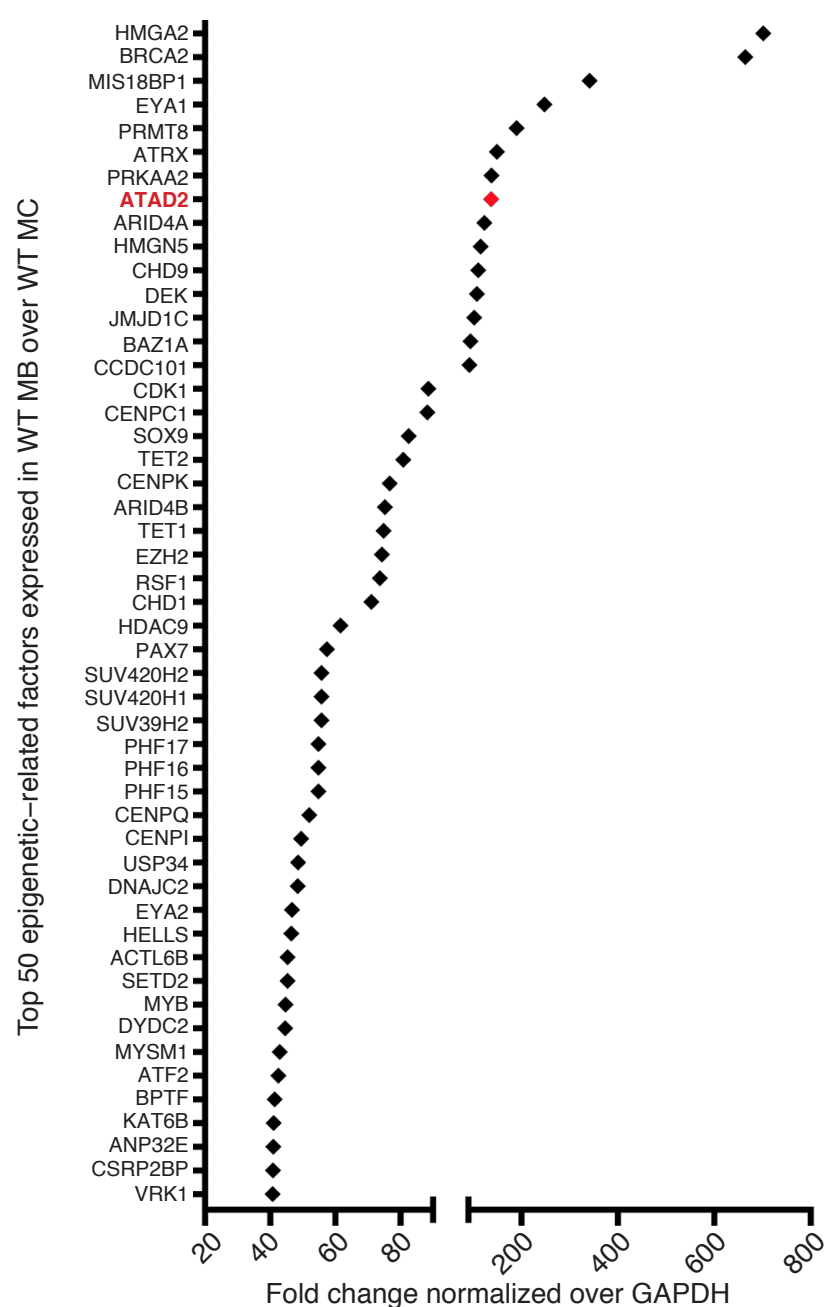
in WT MB versus WT MC
B Expression of epigenetic-related factors

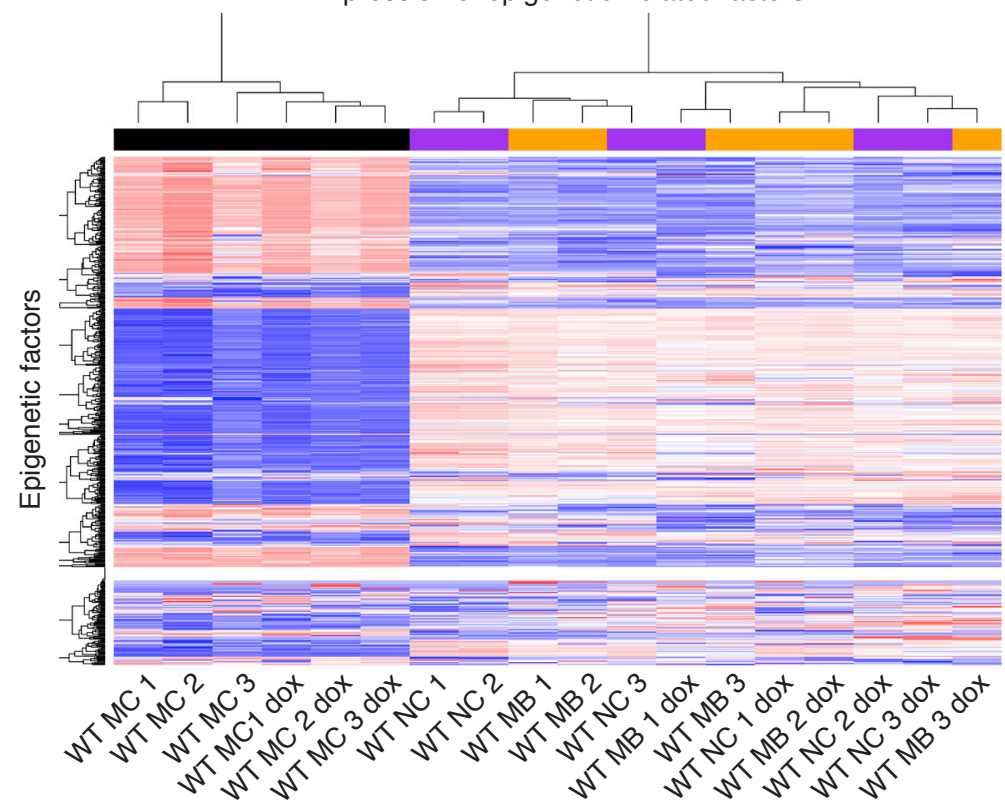

D

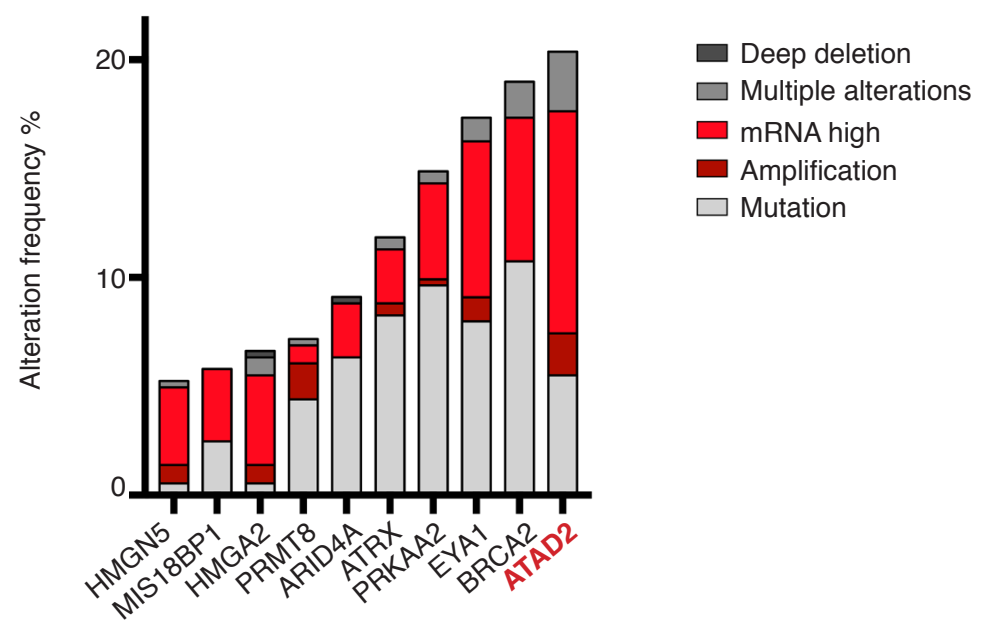

E

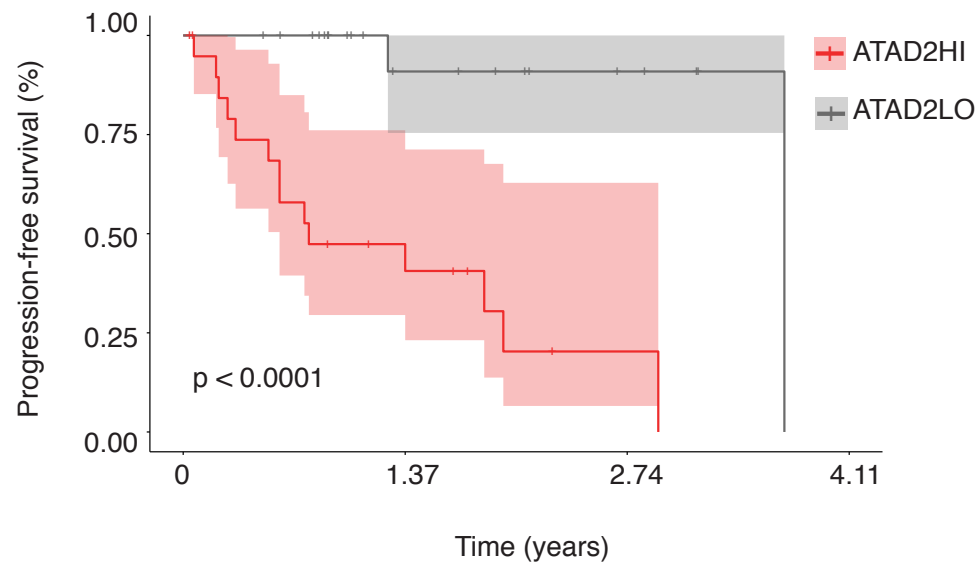


Fig. 3. Cancer competence is reflected by a distinct chromatin landscape and ATAD2 is a key chromatin modifier shared between human NC/MB cells and patient melanoma cells.

(A) GSEA comparing WT MB to WT MC identified chromatin modification as the pathway most enriched in WT MB.

(B) Unsupervised clustering of WT \pm dox NC cells (purple), MB (orange) and MC (black) depending on the expression profile of epigenetic-related factors. WT \pm dox $\mathrm{MC}$ showed a distinct profile from the one of the WT \pm dox NC cells and MB.

(C) Top 50 epigenetic-related factors expressed in WT MB and downregulated in the differentiated WT MC.

(D) Alteration frequency of the top 10 epigenetic-related factors in TCGA SKCM melanoma patient samples.

(E) Kaplan-Meier overall survival curve of TCGA SKCM patients belonging either to the ATAD2HI or to the ATAD2LO group with log-rank p value reported. 
FigbigRxiv preprint doi: $h$ ttps://doi.org/10.1101/2020.05.09.081554; this version posted Male10, 2020. The copyright holder for this preprint (which was not certified by peer review) is the author/funder. All rights resgated. No reuse allowed without permission.

A

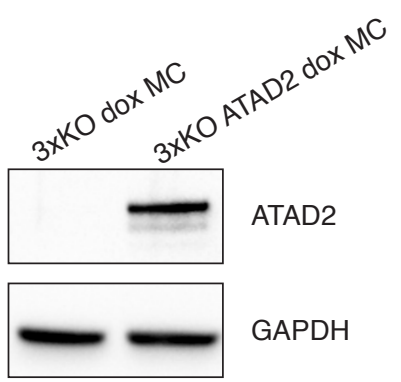

C
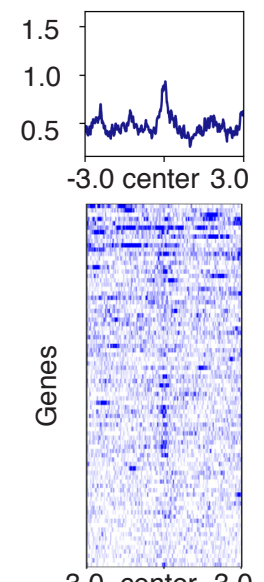

-3.0 center 3.0 gene distance (bp) 3xKO dox MB
NC signature
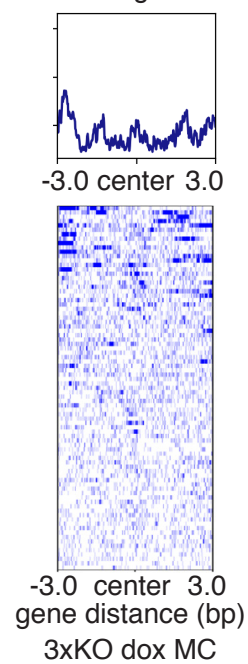

B
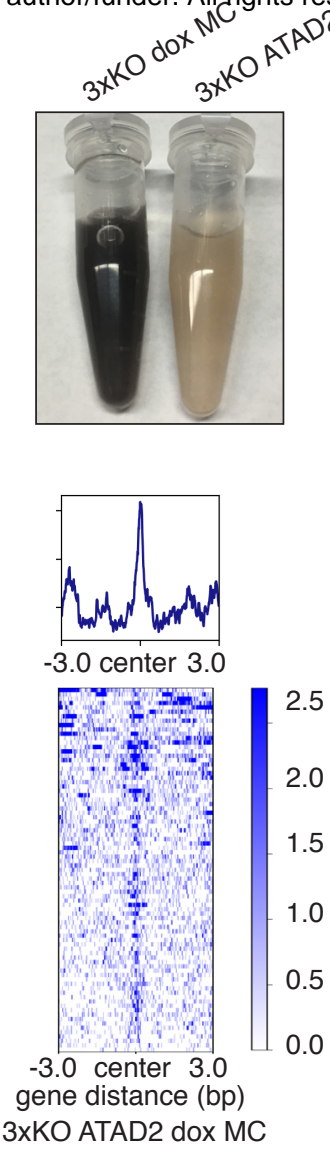

$\underline{\text { SOX10 motif }}$

\section{H}

$\mathbf{F}$

3xKO ATAD2 dox MC vs 3xKO dox MC

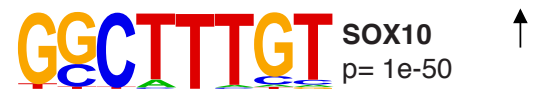

G 3xKO ATAD2 dox MC vs 3xKO dox MC

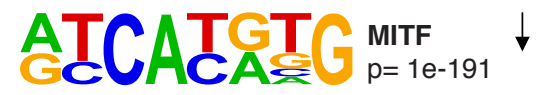

J
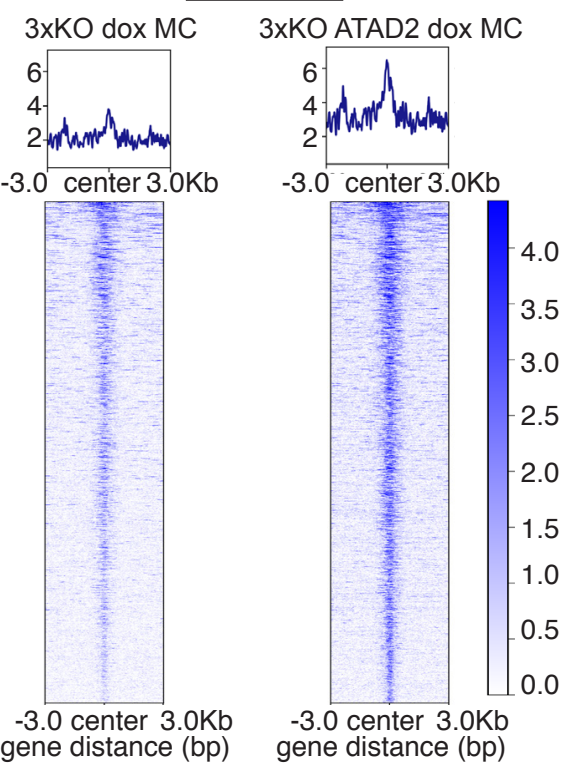
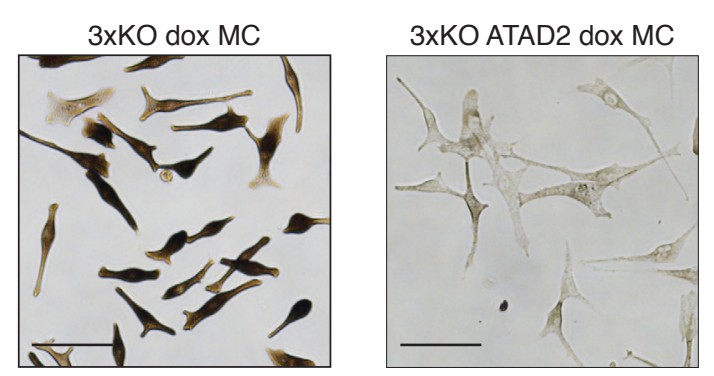

3xKO dox MC vs 3xKO dox MB

D

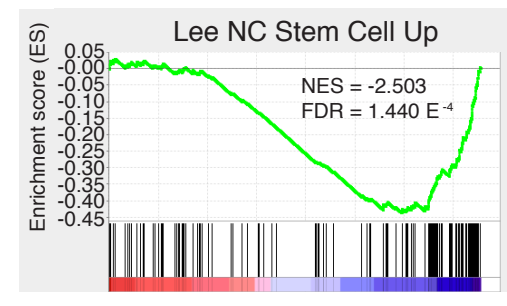

3xKO ATAD2 dox MC vs 3xKO dox MC

E

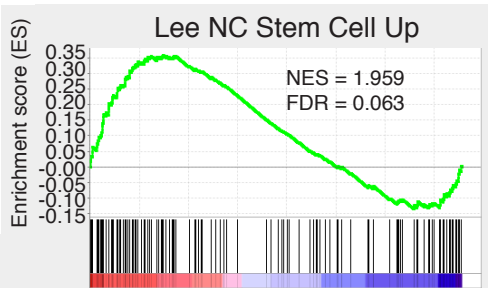

MITF motif

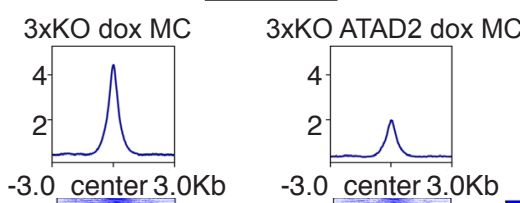

-3.0 center $3.0 \mathrm{~Kb}$
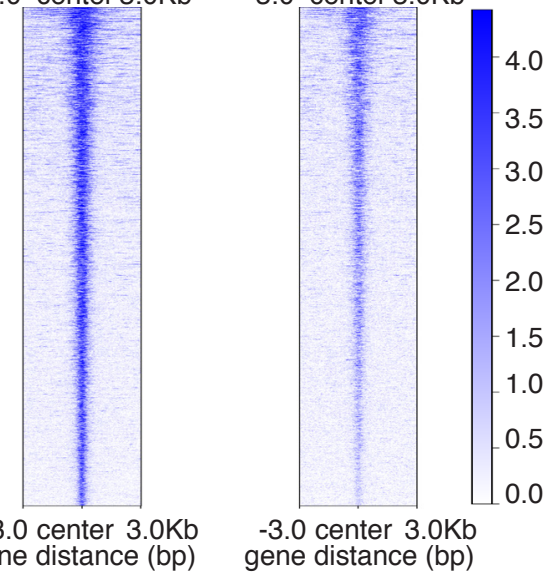

4.0

3.5

3.0

2.5

2.0

1.5

1.0

0.5

-3.0 center $3.0 \mathrm{~Kb} \quad-3.0$ center $3.0 \mathrm{~Kb}$

gene distance (bp) gene distance (bp)

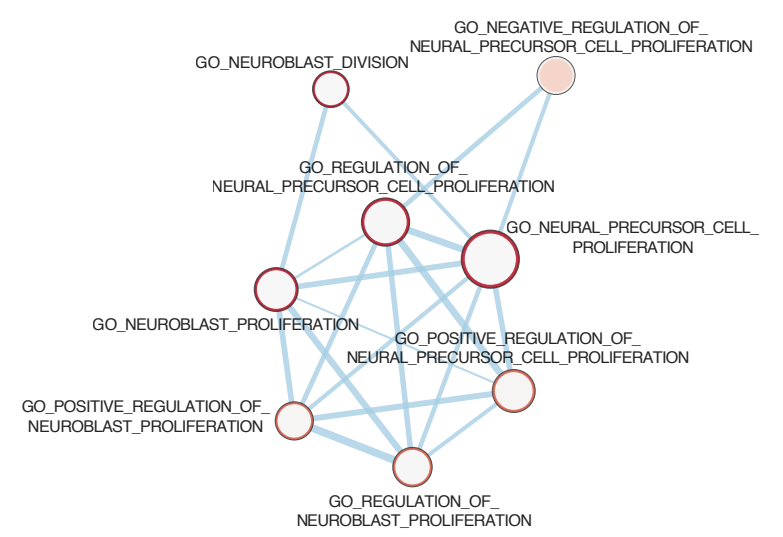

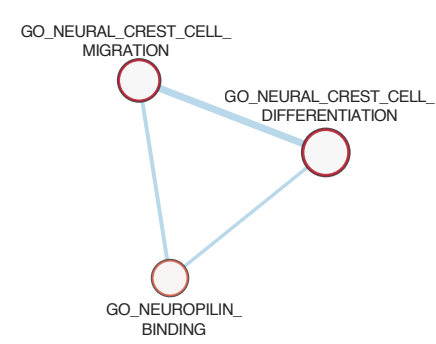

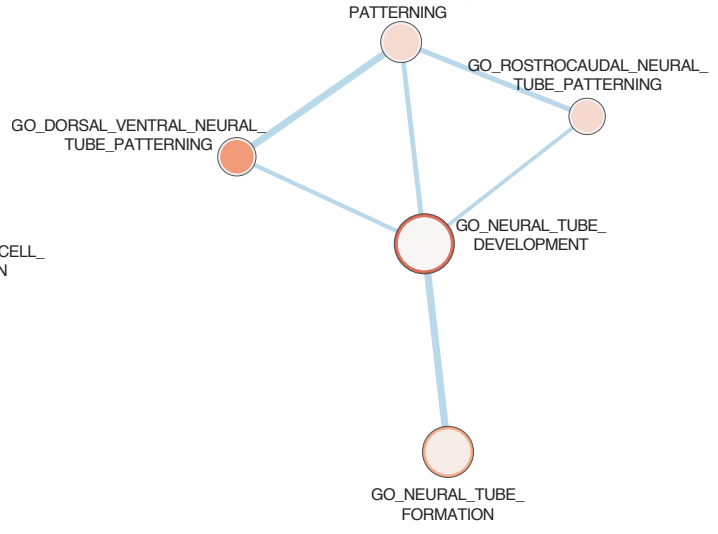




\section{Fig. 4. ATAD2 expression in MC reshapes the chromatin around NC/MB loci and} reactivates a developmental signature.

(A) Western blot for lenti-induced ATAD2 expression in 3xKO dox MC.

(B) Representative pictures of 3xKO dox MC, on the right side, and of 3xKO ATAD2 dox MC, on the left side. Scale bars: $50 \mu \mathrm{m}$.

(C) Tornado plots of the GSEA of the ATAC-seq for genes belonging to the NC signature in 3xKO dox MB, 3xKO dox MC and 3xKO ATAD2 dox MC.

(D-E) GSEA of the ATAC-seq of 3xKO dox MC compared to 3xKO dox MB (D) and of 3xKO ATAD2 dox MC compared to 3xKO dox MC (E).

(F) Homer motif discovery shows that the SOX10 motif is one of the most enriched motifs ( $p$ value $<1 \mathrm{e}^{-50}$ ) in $3 \mathrm{xKO}$ ATAD2 dox MC compared to $3 \mathrm{xKO}$ dox MC.

(G) Homer motif discovery shows that the MITF motif is the most closed motif ( $p$ value $<1 \mathrm{e}^{-191}$ ) in $3 \mathrm{xKO}$ ATAD2 dox MC compared to 3xKO dox MC.

(H) Tornado plots depict the opening of the chromatin specific for the SOX10 motif.

(I) Tornado plots depict the closure of the chromatin specific for the MITF motif.

(J) Network analysis of the genes with increased accessibility for the SOX10 binding motif in 3xKO ATAD2 dox MC compared to 3xKO dox MC. 
Fig:bigRxiv preprint doi: https://doi.org/10.1101/2020.05.09.081554; this version posted May 10, 2020. The copyright holder for this preprint (which was not certified by peer review) is the author/funder. All rights reserved. No reuse allowed without permission.

A

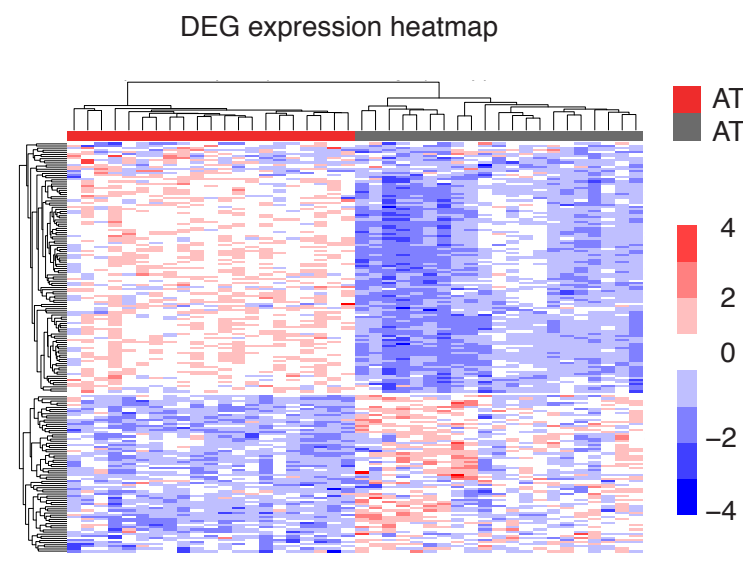

$\mathbf{F}$

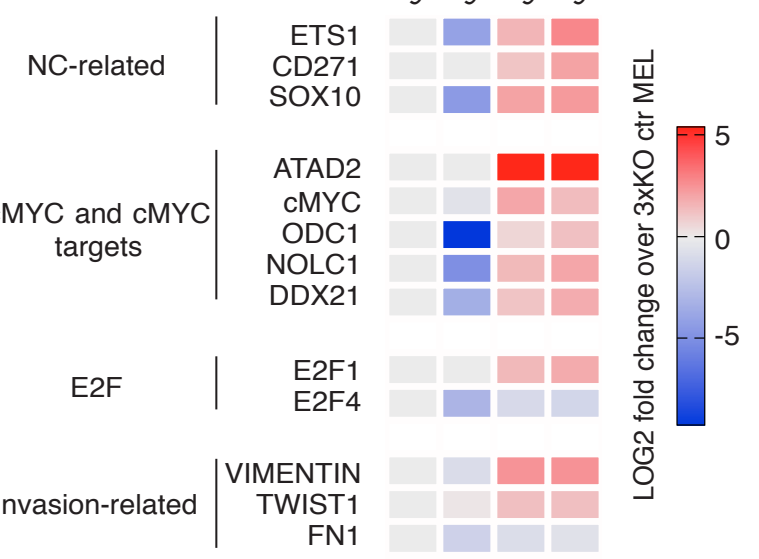

B

Enrichment for genes expressing the SOX motif

ATAD2HI

ATAD2LO

2

C

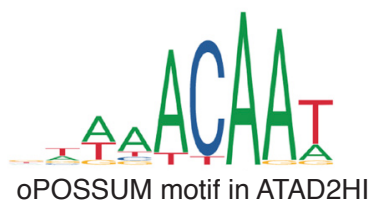

oPOSSUM motif in ATAD2HI ESTROGEN RESPONSE EARLY UV RESPONSE UP

FATTY ACID METABOLISM

Hallmark pathways NES from GSEA

E2F TARGETS

G2M CHECKPOINT

MITOTIC SPINDLE

MYC TARGETS V1 APOPTOSIS MYC TARGETS V2 C TARGETS V 2

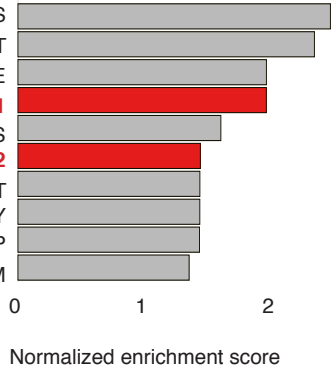

G

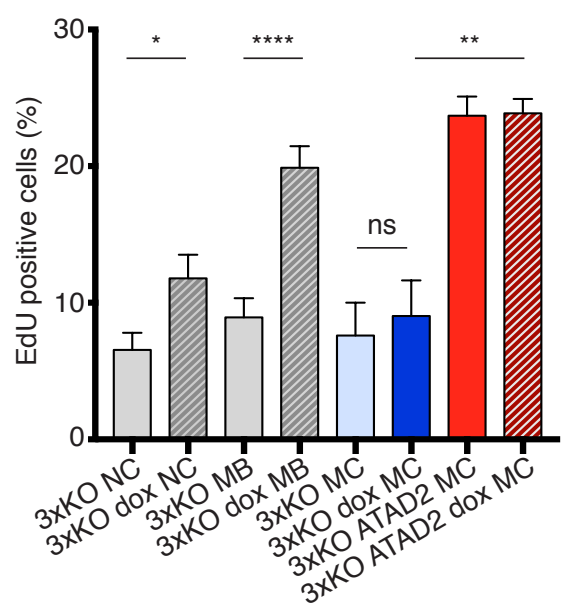

D 3xKO ATAD2 dox MC

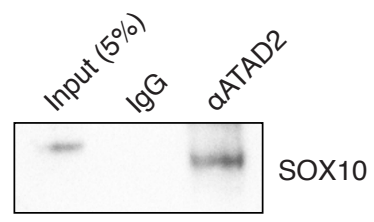

E

3xKO ATAD2 dox MC

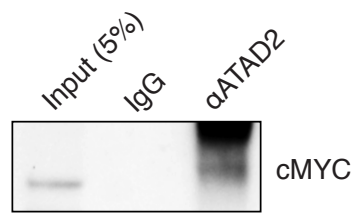

H

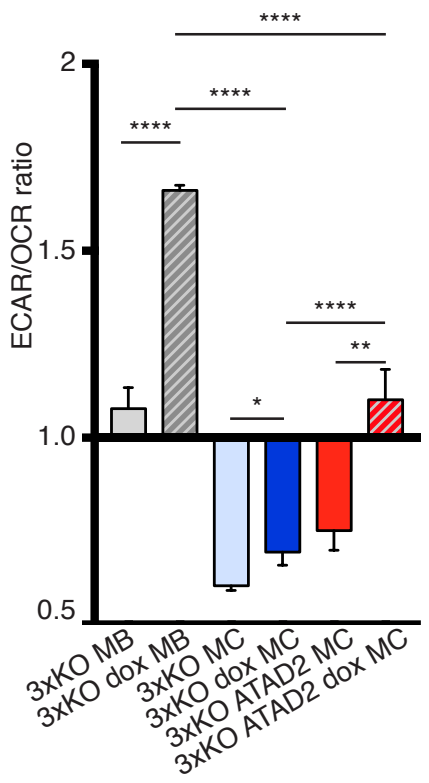




\section{Fig. 5. ATAD2 promotes melanoma phenotypes through $\mathrm{cMYC}$ and SOX10 in} both clinical samples of cutaneous melanoma and in the hPSC-derived cancer model.

(A) Heatmap plot of differentially expressed genes (DEG) in the ATAD2HI patient group versus the ATAD2LO patient group.

(B) Identification of the SOX binding motif on genes co-expressed in the ATAD2HI patient groups, determined by analysis with the oPOSSUM software tool.

(C) Top 10 hallmark pathways from GSEA enriched in the ATAD2HI patient group compared to the ATAD2LO patient group.

(D-E) Co-IP analysis of protein lysates of 3xKO ATAD2 dox MC using either the ATAD2 or the control IgG antibody and then blotted against SOX10 (D) and cMYC (E). (F) qRT-PCR of a subset of NC-related, cMYC-related and invasion-related genes in 3xKO dox MC, 3xKO ATAD2 MC and 3xKO ATAD2 dox MC. Heatmap depicts gene expression changes. $\mathrm{n}=3$ biological replicates. Significance illustrated in the figure refers to comparison to the control $3 \mathrm{xKO} \mathrm{MC}$.

(G) EdU FACS analysis of $3 \mathrm{xKO} \pm$ dox $\mathrm{NC}$ cells, $3 \mathrm{xKO} \pm$ dox $\mathrm{MB}, 3 \mathrm{xKO} \pm$ dox $\mathrm{MC}$ and $3 \times$ KO ATAD2 \pm dox MC upon 30 min of EdU pulse. Data are shown as mean \pm SEM, $\mathrm{n}=3 . *=\mathrm{p}<0.05 ; * *=\mathrm{p}<0.005 ; * * * *=\mathrm{p}<0.0001$

(H) Ratio between the OCR and the ECAR values of $3 \mathrm{xKO} \pm \operatorname{dox} \mathrm{MB}, 3 \mathrm{xKO} \pm \operatorname{dox} \mathrm{MC}$ and $3 x$ KO ATAD2 \pm dox MC. Data are shown as mean \pm SEM, $n=3 . *=p<0.05 ; * *=p$ $<0.005 ; * * * *=\mathrm{p}<0.0001$. 
bioRxiv preprint doi: https://doi.org/10.1101/2020.05.09.081554; this version posted May 10, 2020. The copyright holder for this preprint (which Fig. 6 was not certified by peer review) is the author/funder. All rights reserved. No reuse allowed without permission.

A

TEAZ/Electroporation

MiniCoopR-GFP (to activate BRAF ${ }^{\mathrm{V} 600 \mathrm{E}}$ )

Ub-Cas9

gRB1

Tol2

AltR-Cas9

AltR-sgNT vs AltR-sgATAD2

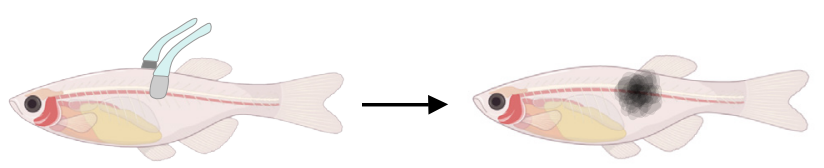

Casper mitfa:BRAF ${ }^{\mathrm{V} 600 \mathrm{E}} \mathrm{p} 53^{-1-}$
B

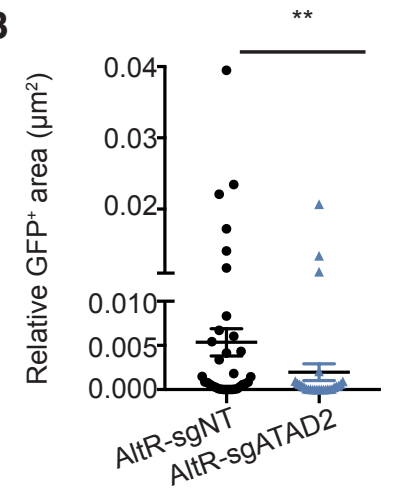

C

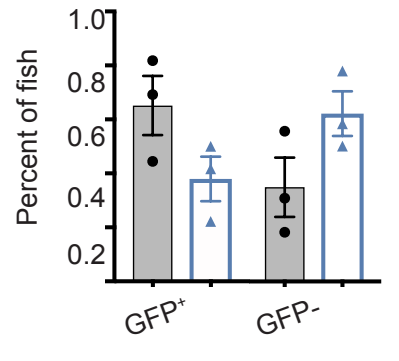

D
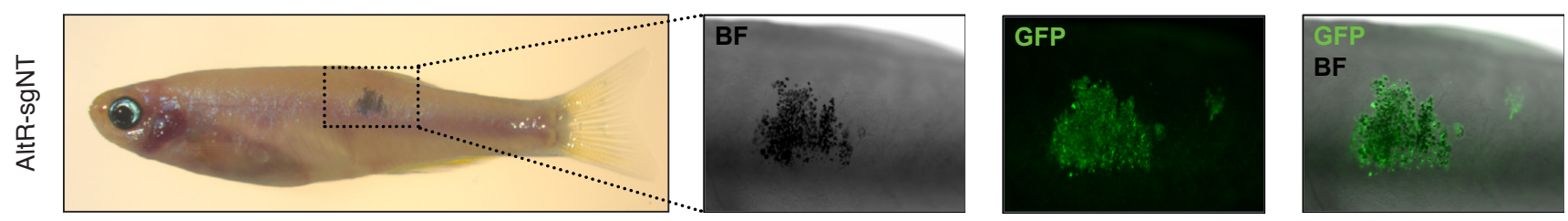

E
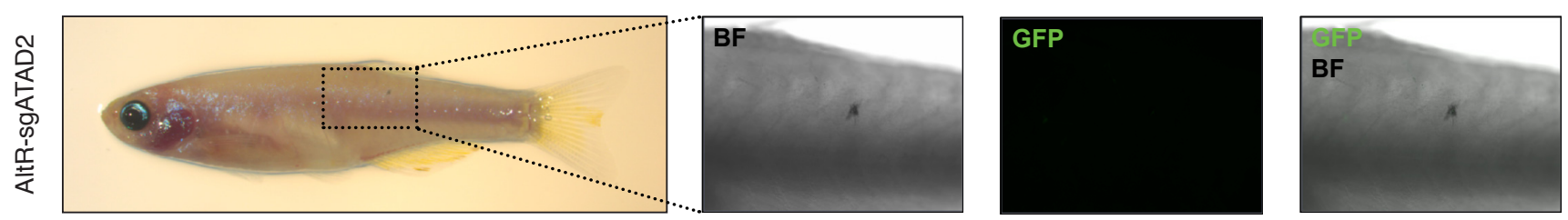

$\mathbf{F}$

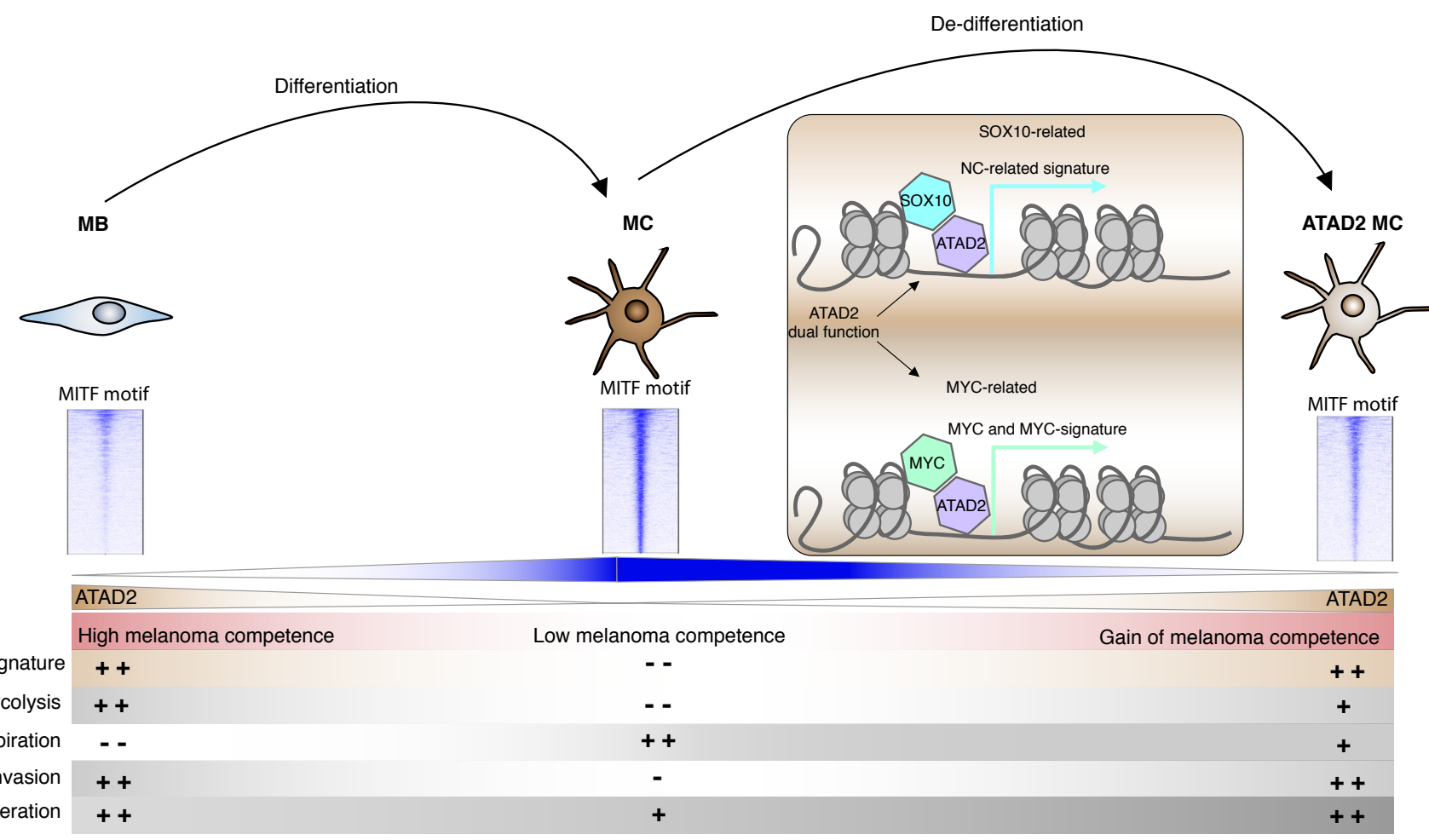




\section{Fig. 6. ATAD2 is required for melanoma initiation.}

(A) Schematic drawing for the TEAZ/Electroporation experiment. Fish that were $\mathrm{p} 53^{-/-}$, mitfa $^{-/-}$, mpv17 $7^{-/-}$and mitfa:hBRAF ${ }^{\mathrm{V} 600 \mathrm{E}}$ were electroporated with MiniCoopR-GFP (mitfa:MITF and mitfa:GFP), Ub-Cas9, gRB1, Tol2, AltR-Cas9, and either AltR-sgNT or a pool of AltR-sgATAD2. The fish were then monitored and quantified for melanoma initiation.

(B) Quantification of the $\mathrm{GFP}^{+}$area $\left(\mu \mathrm{m}^{2}\right)$ in the transgenic fish two weeks after electroporation. Mann-Whitney test with $* * \mathrm{p}=0.01$.

(C) Percentage of fish that were either $\mathrm{GFP}^{+}$or $\mathrm{GFP}^{-}$depending on the electroporation of AltR-sgNT or AltR-sgATAD2.

(D) Representative images of a transgenic fish electroporated with AltR-sgNT. The images depict early lesions characterized by pigmentation and GFP expression.

(E) Representative images of a transgenic fish electroporated with AltR-sgATAD2.

(F) Summary of the roles of ATAD2 in the acquisition of melanoma competence. 